NASA/CR-1998-208674

1N-34

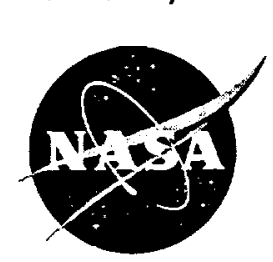

432212

\title{
Direct Numerical Simulation of a Coolant Jet in a Periodic Crossflow
}

Chirdeep Sharma and Sumanta Acharya

Louisiana State University, Baton Rouge, Louisiana 
Since its founding, NASA has been dedicated to the advancement of aeronautics and space science. The NASA Scientific and Technical Information (STI) Program Office plays a key part in helping NASA maintain this important role.

The NASA STI Program Office is operated by Langley Research Center, the Lead Center for NASA's scientific and technical information. The NASA STI Program Office provides access to the NASA STI Database, the largest collection of aeronautical and space science STI in the world. The Program Office is also NASA's institutional mechanism for disseminating the results of its research and development activities. These results are published by NASA in the NASA STI Report Series, which includes the following report types:

- TECHNICAL PUBLICATION. Reports of completed research or a major significant phase of research that present the results of NASA programs and include extensive data or theoretical analysis. Includes compilations of significant scientific and technical data and information deemed to be of continuing reference value. NASA's counterpart of peerreviewed formal professional papers but has less stringent limitations on manuscript length and extent of graphic presentations.

- TECHNICAL MEMORANDUM. Scientific and technical findings that are preliminary or of specialized interest, e.g., quick release reports, working papers, and bibliographies that contain minimal annotation. Does not contain extensive analysis.

- CONTRACTOR REPORT. Scientific and technical findings by NASA-sponsored contractors and grantees.
- CONFERENCE PUBLICATION. Collected papers from scientific and technical conferences, symposia, seminars, or other meetings sponsored or cosponsored by NASA.

- SPECIAL PUBLICATION. Scientific, technical, or historical information from NASA programs, projects, and missions, often concerned with subjects having substantial public interest.

- TECHNICAL TRANSLATION. Englishlanguage translations of foreign scientific and technical material pertinent to NASA's mission.

Specialized services that complement the STI Program Office's diverse offerings include creating custom thesauri, building customized data bases, organizing and publishing research results ... even providing videos.

For more information about the NASA STI Program Office, see the following:

- Access the NASA STI Program Home Page at http://www.sti.nasa.gov

- E-mail your question via the Internet to help@sti.nasa.gov

- Fax your question to the NASA Access Help Desk at (301) 621-0134

- Telephone the NASA Access Help Desk at (301) 621-0390

- Write to: NASA Access Help Desk NASA Center for AeroSpace Information 7121 Standard Drive Hanover, MD 21076 
NASA/CR-1998-208674

\section{Direct Numerical Simulation of a Coolant Jet in a Periodic Crossflow}

Chirdeep Sharma and Sumanta Acharya

Louisiana State University, Baton Rouge, Louisiana

Prepared under Grant NAG3-1641

National Aeronautics and

Space Administration

Lewis Research Center 


\section{Acknowledgments}

This work was supported by an AST grant from the Turbomachinery Fluid-Physics group of NASA Lewis with Dr. Chi Wang and Dr. Ray Gaugler serving as monitors. This support is gratefully acknowledged.

We also appreciate the help of Professor Joel Tohline of the Physics Department at LSU for providing us with computer time to run on the MasPar computing system.

Available from

NASA Center for Aerospace Information 7121 Standard Drive

Hanover, MD 21076

Price Code: A04
National Technical Information Service 5285 Port Royal Road Springfield, VA 22100 Price Code: A04 


\title{
Direct Numerical Simulation of a Coolant Jet in a Periodic Crossflow
}

\author{
Chirdeep Sharma' and Sumanta Acharya' \\ Mechanical Engineering Department \\ Louisiana State University \\ Baton Rouge, LA 70803
}

\begin{abstract}
A Direct Numerical Simulation of a coolant jet injected normally into a periodic crossflow is presented. The physical situation simulated represents a periodic module in a coolant hole array with a heated crossflow. A collocated finite difference scheme is used which is fifth-order accurate spatially and second-order accurate temporally. The scheme is based on a fractional step approach and requires the solution of a pressure-Poisson equation. The simulations are obtained for a blowing ratio of 0.25 and a channel Reynolds number of 5600 . The simulations reveal the dynamics of several large scale structures including the Counter-rotating Vortex Pair (CVP), the horse-shoe vortex, the shear layer vortex, the wall vortex and the wake vortex. The origins and the interactions of these vortical structures are identified and explored. Also presented are the turbulence statistics and how they relate to the flow structures.
\end{abstract}

\section{Introduction}

In recent years, Direct Numerical Simulation (DNS) has become a powerful tool in understanding flow physics and the interaction between various dynamical scales in the flow. Numerical Simulation of channel flows, due to its geometrical simplicity, has been studied most commonly in the DNS literature. Orszag et al. (1980) studied transition from laminar to turbulent

\footnotetext{
'Graduate Student

${ }^{2}$ Professor and author to whom all correspondence should be addressed
} 
flow using a 3-step time splitting method. Fourier series expansions in the spanwise and streamwise directions and Chebysher polynomial expansion in the direction normal to the walls. Orszag et al. (1983) later used the same algorithm. but with an explicit Adams-Bashforth integration scheme, to perform a DNS of turbulent channel flow. A DNS for a curved channel flow was carried out by Moser et al. (1984) at $\mathrm{Re}_{i}=180$ again using a spectral method. Kim et al. (1987) used a Fourier Chebyshev pseudospectral method to study fully turbulent channel flow at the same Reynolds number and presented a large number of turbulence statistics. A finite difference DNS for a turbulent channel flow was presented by Rai et al. (1989) who showed the need for higher order accurate upwind schemes to yield accurate simulations.

These initial successes with the DNS method spurred the investigation of slightly more complex situations. Gavrilakis (1992) and Huser and Biringen (1993) both reported DNS for flow in a square duct geometry. Lyons et al. (1991) studied fully developed passive heat transfer in a direct simulation based on spectral method. Kasagi et al.(1992) and Kim and Moin (1989) obtained the solution to the energy equation for channel flows using a DNS procedure. Sumitani and Kasagi (1995) have reported a DNS in a channel flow with uniform suction and injection. More recently, Pointel et al (1996) have presented a DNS of channel flow with heat transfer using a collocated fifth-order accurate finite difference procedure. The calculations were done for a $\operatorname{Re}_{\tau}=180$ on a $128 \times 64 \times 64$ grid and were found to be in good agreement with the DNS data of Kim et al. (1987) and the measurements of Kreplin and Eckelmann (1979).

The present paper aims to extend the work of Pointel et al. (1996) for a more complex flow situation, and intends to perform a DNS of a periodic cross-flow in a channel with a vertical 
slot-injection of coolant through the bottom surface. Examples of slot injection into a crossflow include the film cooling of gas turbine blades and combustor walls, flow control of aircraft and missiles through blowing, and effluent discharges into air or water bodies. The application of primary interest here is the film cooling of gas turbine blades.

Jets in crossflow, as encountered in film cooling configurations, represent a rather complex flow situation. Several investigators have studied the features of the flow field associated with a single jet in a crossflow. The counter rotating vortex pair (CVP) is the most dominant structure observed, and persists far downstream of the jet injection. The CVP is a consequence of the reorientation of the jet-hole vorticity by the crossflow (Foss (1980), Andreopoulos (1985), Kelso et al. (1996)). Shear layer or ring vortices have also been observed in the near field of the jet. These vortices are driven by the Kelvin-Helmoltz instability. Horseshoe vortices are produced as a consequence of the jet obstructing the crossflow producing an adverse pressure gradient just upstream of the jet. These vortices have spanwise vorticity when they form, and as they travel downstream they are deflected by the crossflow and acquire streamwise vorticity rotating in a direction opposite to the CVP. Wake vortices which exist between the wall and the jet have also been observed (Fric and Roshko, 1994).

The majority of the studies aimed at numerically examining the details of the flow structures have approached the problem by solving the Reynolds Averaged Navier Stokes (RANS) equations. This time-averaged approach requires the use of turbulence models and makes predictions of the dynamic nature of vortices unfeasible. RANS calculations have been reported by Patankar et al. (1977), Sykes et al. (1986), Kim and Benson (1992) and Demuren (1993). Recently Large Eddy Simulations (LES) that resolve the dynamics of the large scales only and 
model the small scales have been presented by Jones (1996) and Yuan and Street (1996).

The geometrical configuration of interest considered in this paper is shown in Figure 1. The primary flow (called the crossflow) in the channel is in the $x$-direction. The coolant jet is injected vertically upwards from a rectangular slot on the bottom surface. Periodic boundary conditions are imposed on the crossflow in the $\mathrm{x}$-direction. The periodic flow in the $\mathrm{x}$-direction makes the computational problem not only simpler to handle but it also allows us to mimic the effects of upstream film coolant holes. In a film cooling configuration with a series of parallel holes in the streamwise direction, the downstream coolant hole will see the downwash from the upstream coolant hole, and this effect is incorporated in the present configuration with the streamwise periodic boundary condition. Single coolant hole studies with Dirichlet inflow and outflow conditions can not simulate the upstream hole effects. The correct alternative is to include all rows of holes in the calculation domain, but performing a DNS calculation for such a configuration is considerably more difficult than the simpler single-hole situation considered in this paper. However, for a single hole domain, the imposition of periodic boundaries in the crossflow direction is inappropriate, unless a suction boundary condition is incorporated, since with injection alone there is a net addition of mass across the lower surface. Therefore, in order to impose periodic conditions in the primary flow direction, a slot-suction boundary condition is imposed on the top wall. The normal suction velocity and temperature at the top wall is made exactly equal to the normal injection velocity and temperature at the bottom wall so that the mass, momentum and energy injected in exactly balances that sucked out. The blowing (or suction) ratio (injection velocity/crossflow velocity) is taken to be sufficently small (0.25) so that the injection and suction effects are primarily confined to a small distance normal to the surface. 
In the spanwise direction, a row of film coolant holes are assumed to be present. Therefore, in the computational domain of this study. consisting of a single hole, periodic spanwise boundary conditions are imposed.

\section{Numerical Procedure}

Assuming constant density, and using the channel half-width $\delta$ as the length scale, and the friction velocity $u_{\tau}$ for the fully-developed channel flow (with no injection or suction) as the velocity scale, the non-dimensional continuity equation and the Navier Stokes equation for each velocity component $u_{i}(i=1,2$ and 3 represent the $u, v, w$ velcoity components) can be written as:

$$
\begin{gathered}
\vec{\nabla} \cdot \vec{u}=0 \\
\frac{\partial u_{i}}{\partial t}+(\vec{u} \cdot \vec{\nabla}) u_{i}=-\vec{\nabla} p+\frac{1}{\operatorname{Re}_{\tau}} \nabla^{2} u_{i}
\end{gathered}
$$

In the above equation, $\mathrm{p}$ represents the pressure and $\mathrm{Re}_{\tau}$ represents the Reynolds number based on the fully-developed channel flow friction velocity. For the temperature, iso-flux, iso-thermal conditions proposed by Kasagi et al. (1992) have been adopted. According to their investigations, for iso-flux conditions, the variations in the wall temperature for air flow can be considered to be negligible. Hence we can assume the wall to be locally isothermal. The isoflux condition implies that the ensemble averaged wall temperature (over $z$ and $t$ ), increases linearly with $x$-direction. Hence the bulk mean temperature averaged over $\mathrm{z}$ and $\mathrm{t}$ also increases linearly in $\mathrm{x}$-direction and can be obtained from an energy balance. We also assume that the injection and suction jets are at the same temperature but lower than that of the wall. Hence the overall energy balance is 
preserved. The non-dimensional energy equation can then be expressed as:

$$
\frac{\partial \theta}{\partial t}+(\vec{u} \cdot \vec{\nabla}) \theta=\frac{1}{\operatorname{Re}_{\tau} \operatorname{Pr}} \nabla^{2} \theta+\frac{2}{\int_{\cdot 1}^{1} \bar{u} d y}
$$

where

$$
\theta(x, y, z, t)=\frac{\left(T_{w}\right) T(x, y, z, t)}{T_{\tau}}
$$

and $T_{\tau}$ is the friction temperature given by $q_{w} /\left(\rho c_{p} u_{\tau}\right)$, and $q_{w}$ and $\left\langle T_{w}\right\rangle$ are the ensemble averaged heat flux and wall temperature (over $z$ and $t$ ) respectively.

Results are obtained for $a \operatorname{Re}_{\tau}=180$. This corresponds to a channel Reynolds number $\operatorname{Re}_{\mathrm{m}}$ based on the mean velocity and the channel height of 5600 . As noted earlier, DNS results for fully developed channel flow at this $\mathrm{Re}_{\tau}$ value were reported by $\mathrm{Kim}$ et al. (1987) and Pointel et al. (1996). Since in the configuration considered in this study, the net mass addition is zero, $\operatorname{Re}_{\tau}=180$ represents an average value for the channel. The injection and suction jet-holes were placed in the center of the lower and upper walls and were rectangular in shape with the long dimension of the hole (d) along the crossflow direction (x) and the short dimension of the hole (w) in the spanwise direction (z). The corresponding non-dimensional hole sizes $d / \delta$ and $w / \delta$ were 0.5 and 0.2 respectively. The actual non-dimensional lengths of the computational domain were chosen to be $4 \pi \times 2 \times 0.4 \pi$. These correspond with the $4 \pi \times 2 \times 2 \pi$ computational domain dimensions of Pointel et al. (1996), except in the spanwise direction where coolant hole spacings of $2.5 \mathrm{~d}(=1.25=0.4 \pi$ in non-dimensional units) has been assumed. 
As noted earlier. the flow and temperature variables are assumed to be periodic in the $\mathrm{x}$ and $z$ directions. Dirichlet. zero velocity and temperature, boundary conditions were imposed at the channel walls while a zero-value Neumann condition was imposed on the nomal pressure gradient at the wall. At the injection and suction slots, the normal velocity is set equal to 0.25 of the mean crossflow velocity corresponding to the fully developed channel flow at $\operatorname{Re}_{\tau}=180$, and the temperature is set to a value lower than the wall temperature, and is assigned a nondimensional value of 0.5 .

The Fractional Step approach originally developed by Chorin (1967) was used for integrating the equations. In the first step, the momentum equation without the pressure gradient contribution is solved using a second order explicit Adams-Bashforth time-integration for the convection and streamise and spanwise diffusion terms. For the normal-diffusion term, an implicit Crank-Nicolson scheme is used. This approach uses an intermediate velocity (denoted by $\mathrm{a}^{-}$), and for a typical velocity component, say $\mathrm{u}$, has the following form:

$$
\frac{\tilde{u}-u^{n}}{\Delta t}=\frac{3}{2}\left(C^{n}+D_{e}^{n}\right)-\frac{1}{2}\left(C^{n-1}+D_{e}^{n-1}\right)+\frac{1}{2}\left(D_{i}^{n}+\widetilde{D}_{i}\right)
$$

Here $C$ represent the convection terms, $D_{c}$ represents the streamwise and spanwise diffusion terms and $D_{i}$ represents the normal diffusive term.

The velocity field is computed in the second step:

$$
\frac{u^{n+1}-\tilde{u}}{\Delta t}=-\nabla \phi^{n+1}
$$


where the pseudo pressure $\phi$ is related to the pressure by:

$$
\phi-\frac{\Delta l}{2 \operatorname{Re}} \frac{\partial^{?} \phi}{\partial y^{2}}=p
$$

We can apply the divergence operator on equation (5) and use continuity of velocity field to obtain the pressure Poisson equation:

$$
\frac{1}{\Delta} \nabla \tilde{u}=\nabla \cdot\left(\nabla \phi^{n+1}\right)
$$

Once the pseudo pressure is known the velocity field $\mathrm{u}^{\mathrm{n}+1}$ can be calculated from equation (5).

The scalar field for temperature is then obtained by integration of the energy equation. We use a similar approach to the momentum equation to get the fractional step equations from which we can directly obtain the temperature field.

The spatial discretization was done using the non-conservative formulation of the convective and diffusive terms on a collocated grid with velocity and pressures at the center of the control volume. The convective terms were treated by a fifth order upwind biased approximation as used by Rai et al. (1989). The diffusive terms are treated by a sixth order accurate seven point central difference stencil. For the boundary terms we used one sided differences to maintain the accuracy of the scheme. The pressure Poisson equation is discretized using the 4-2 consistent formulation as recommended by Najjar et al. (1994). This involves second order discretization of the divergence operator and fourth order discretization of the pressure gradient operator. In the continuity equation, which is used for updating the velocity field at the cell faces, the gradient operator is represented by a fourth order finite difference formulation. 
The implementation of the numerical procedure, described below, is done on a MasPar MP-2. This is a massively parallel computer and provides a suitable platform for computer intensive DNS calculations. The MasPar is a SIMD machine, which has a $128 \times 64$ parallel processors (PE) array. We have used a grid of $128 \times 64 \times 64$ for the calculation, which is uniform in the $\mathrm{x}$ and $\mathrm{z}$ direction, and non-uniform in the $\mathrm{y}$ direction. Each $(I, J)$ node is mapped on to a PE. Each PE has a local memory of $64 \mathrm{~K}$ and provides the storage in the third $(\mathrm{K})$ dimension.

The time integration was first done for the velocity field till the flow reached a fully developed state. Then the thermal field was allowed to reach the fully developed state before the calculation of mean quantities was started. Typically 60,000 time steps were required to get to this stage. The calculation of the mean quantities was done over 20,000 non-dimensional time steps. This was followed by the calculation for the turbulent statistics and this was done over 8000 steps. During the statistics calculation we also calculated the instantaneous vorticity profiles for various streamwise, crossstream and spanwise planes at various time steps in order to present the time evolving characteristics for the flow field.

\section{Results and Discussion}

In making an assessment of the adequacy of the grid size used in the present study where the mean $\mathrm{Re}_{\tau}$ is 180 , we make comparisons with the grid resolution of reported channel flow studies at a $\mathrm{Re}_{\imath}$ of 180 . Pointel et al. (1996) used a $128 \times 64 \times 64$ grid for simulating fully developed channel flow in a $4 \pi \times 2 \times 2 \pi$ domain at a $\operatorname{Re}_{\mathrm{t}}$ of 180 . Results obtained are shown in Figure 2 and compared with the DNS predictions of Kim et al. (1987) and the experimental data of Kreplin 
and Eckelmann (1979). As can be seen, the comparison is quite good. The same $128 \times 64 \times 64$ grid was employed in the present problem which has a smaller computational domain of $4 \pi \times 2 \times$ $0.4 \pi$, and therefore has an improved resolution. The resolution in wall-coordinates are noted to be: $\Delta x^{+}=17.67, \Delta y^{+}=0.018-23.76, \Delta z^{+}=3.59$. In comparison, Kasagi et al. (1992) reported corresponding grid spacings of $18.4,0.08-4.9$ and 7.4 in a $5 \pi \times 2 \times 2 \pi$ computational domain. Similar grid spacings of 17.7, 0.05-4.4, and 5.9 were also used by Kim and Moin (1989) and Kim et al. (1987) in a $4 \pi \times 2 \times 2 \pi$ computational domain at the same Reynolds number. Moser and Moin (1984) used $128 \times 65 \times 128$ points in a similar computational domain and comparable Reynolds number, and reported that this resolution was adequate to resolve all the essential turbulent scales. Lu and Hetsroni (1995), in their direct simulation of channel flow with heat transfer at a comparable Reynolds number, have used $\Delta \mathrm{x}^{+}, \Delta \mathrm{y}^{+}$, and $\Delta \mathrm{z}^{+}$values of $32,0.4-20$, and 8. Based on these comparisons, the grid size used in this study should be deemed to be adequate.

In presenting the results below, the origin of the coordinate system is fixed at the jet-hole center. The $x$ and $y$ dimensions are scaled with the long dimension of the jet hole (d) oriented in the direction of the crossflow, while the $\mathrm{z}$ dimension is scaled with the spanwise dimension of the jet hole (w).

\section{Characteristics of the Flowfield}

Figures 3(a) and (b) present the $u$ and $v$ velocity contours in the spanwise midplane $(z / w$ $=0$ ), and correspond to the vector plots shown in figures $5(\mathrm{a})$ and $5(\mathrm{~b})$. For clarity, several of the

plots are stretched in the vertical or spanwise directions. Figure 3(a) and 5(a) clearly show the 
almost immediate bending of the jet due to the strong crossflow. The jet penetration is confined to a $y / \delta$ of about 0.5 or a $y / d$ of about 1 which corresponds to the long-dimension of the jet-hole (hereinafter referred to as the jet diameter). Figure 5a clearly shows that at the channel mid-plane $(y / d=2)$, the crossflow velocity is relatively unaffected by the injection or suction slots. This observation supports the earlier premise that the behavior of the film-coolant injection jet can be studied independent of the suction jet, and that the observations made should hold for a film cooling configuration with a series of coolant holes in the crossflow (or primary flow) direction.

A weak horseshoe vortex system is observed in Fig. 3(a) and in the near-wall detail of the velocity vectors shown in Fig. 5(b). The vortex system extends over one hole diameter upstream of the jet and appears to consist of two clockwise eddies rotating side by side. However, the horse-shoe vortex system does not appear to be deflected around the jet as it does at higher blowing ratios (see Fric and Roshko, 1994; Kelso et al., 1996; Muldoon and Acharya, 1998). No footprint of the vortex is seen in the $x$-vorticity contour along a horizontal plane just above the lower surface (Fig. 3c).

In examining the instantaneous v-velocity contours (Fig. 3b), it can be seen that roughly 5 hole diameters downstream of the injection location $(x / d=5)$, altemate pockets of positive and negative v-contours are present (note the high level of stretching in the $y$-direction). These are analogous to those observed by Kim et al. (1987), and represents ejection and sweep events associated with a developing boundary layer. In the near vicinity of the injection hole, $1<x / d<$ 5 , the behavior is different with primarily positive near wall velocities.

The $x$-vorticity contours shown in Figure $3 c$ indicate the existence of a dominant wall- 
vortex structure close to the lower surface with vorticity opposite to the CVP. Thus downstream of the injection hole, positive vorticity is seen close to the wall in the right half (viewing the flow from a downstream point) and negative vorticity is noted in the left half. Further away from the wall, at a higher $y / d$ value, the CVP is encountered and the sign of the vorticity reverses. This can be seen in the cross-sectional $(y-z)$ plots of $x$-vorticity presented later. Figure $3 c$ also shows the generation of negative and positive $x$-vorticity on the right and left edges of the jet hole; these represent the source of vorticity for the CVP. It is however important to note, that the vorticity for the CVP does not originate from any in-hole boundary layer vorticity (as has often been reported in the literature), since at the jet exit plane a uniform v-velocity was specified. Rather, the x-vorticity along the jet-hole edges is generated by the interaction of the injected velocity and the crossflow.

Figure 4 shows the velocity vectors in different cross-stream planes at $x / d=0.5,2,5$ and 10. At $x / d=0.5$ we can see the jet penetration to be more than $y / d=1.8$. However beyond a $y / d$ of 1 , the velocity magnitudes in the $y-z$ plane are considerably smaller than the velocities in the $x$ y plane, and therefore as seen in Fig. Sa, the flowfield is relatively unaffected by the jet beyond a $y / d$ of 1 . We can see the evolution of the CVP around $z / w= \pm 0.8$ for $y / d<0.2$. There is considerable flow across the spanwise periodic boundaries indicating a significant level of lateral transport, and interaction between spanwise adjacent coolant jets. Both inflow and outflow are noted across the periodic boundaries. We also note the absence of any symmetry across the $z / w=0$ boundary. In fact by $x / d=10$, the CVP has lost its bound-vortex structure and no semblance of symmetry exists across $z / w=0$. In the near field $(x / d<5)$, each half of the CVP is 
clearly associated with a weak counterrotating eddy system above it.

Figure $5 \mathrm{c}$ shows that there is a significant amount of entrainment of the crossflow into the wake of the jet. This clearly appears to persist up to about $x^{\prime} \delta$ of 9 at which point the wake appears to meander away from the jet-hole centerline. A close inspection also reveals flow reversals of the entrained crossflow near the jet-hole centerline. These reversals are most clearly seen between $\mathrm{x} / \mathrm{d}$ of 3 and $5 \mathrm{in} \mathrm{Fig.} \mathrm{5c,} \mathrm{and} \mathrm{represent} \mathrm{the} \mathrm{onset} \mathrm{of} \mathrm{the} \mathrm{wall-vortex} \mathrm{system} \mathrm{which}$ will be clearly evident in the $x$-vorticity contours in a $y$-z plane presented later.

Figure 6 shows the profiles of the mean velocity and temperature, non-dimensionalized by the corresponding average friction-velocity $u_{\tau}$ (which is the same as the friction velocity for fully developed turbulent channel flow at the same average Reynolds number $\operatorname{Re}_{\tau}=180$ of this study) and the average friction-temperaure $T_{\tau}$. The profiles are presented for several $x / d$ locations and two $z / w$ planes, $z / w=0$ corresponding to the jet-center plane, and $z / w=-1.9$ representing a location between the jet-hole and the spanwise periodic boundary. The near-wall behavior $\left(y^{+}=y \cdot \operatorname{Re} / \delta<12\right)$, appears to conform somewhat to the law-of-the-wall behavior with a maximum deviation of less than $30 \%$. However, in the turbulent region $\left(y^{+}>12\right), \log$-law behavior is not observed even off the jet-center plane and upstream of the jet $(z / w=-1.9, x / d=-2)$ due to the effect of vortical flow structures. At $z / w=-1.9$, the near-wall behavior appears to be only mildly altered by the spanwise cross-stream flow downstream of the jet In fact, Fig. $5 \mathrm{c}$ indicates that the spanwise cross-stream flow close to the wall at $z / w=-1.9$ is relatively weak. The maximum deviation from the $x / d=-2$ profile appears to occur in the vicinity of $x / d=2$, beyond which flow recovery is initiated. The profiles in the jet-center plane (Fig. $6 \mathrm{~b}$ and $6 \mathrm{~d}$ ) indicate significant 
distortion of the near-wall behavior by the jet This distortion begins at the upstream end of the jet $\left(x^{\prime} d=-0.5\right)$ with a significant reduction in the wall-gradients. Flow recovery begins dounstream of the jet, but the recovery in the inner-region $\left(y^{+}<12\right)$ is substantially faster than in the outerregion $\left(y^{+}>12\right)$, leading to a change in the sign of the slope in the velocity profile in the buffer region and immediately above it $\left(7<y^{*}<35\right.$ or $\left.0.08<y / d<0.39\right)$. Such changes in the slope of the velocity profile can lead to the development of flow instabilities and large-scale vortical structures. This $y / d$ region can be seen to correlate with the region associated with the growth of large scale structures in the vorticity contours presented later.

Figure 7 shows the time history of spanwise vorticity at $z / w=0$. The time-series is presented at time instances $t_{1}, t_{2}, \ldots t_{8}$, separated by $\Delta t=0.02$ where $\Delta t$ is the non-dimensional time interval. The figures indicate the predominant negative $\omega_{2}$ on the windward side which is indicative of the crossflow bending due to the jet. Patches of positive vorticity can be seen on the leeward side of the jet. The vortices on the windward and leeward side of the jet are driven by Kelvin-Helmholtz instabilities, and are generally referred to as shear layer or spanwise-jetvortices. These vortices, particularly those from the windward side, appear to be peeled by the crossflow and convected downstream. In fact the near-wall boundary-layer region downstream of the jet is dominated by the negative vorticity partly originating from the windward side of the jet, and partly associated with a developing boundary layer. The dynamical nature of the vortices should be noted in the time-series presented. Note the vortices on the windward side at time $t_{3}$ between $y / d$ of 0.5 and 1. At the next time-instance, a necking process is initiated, and subsequently two vortices form and convect downstream. A third vortex is transported to the 
near wall region dounstream of the jet. Upstream of the jet the horseshoe vortex system consisting of two eddies can be observed. but it can be seen to be rather small and localized.

Figure 8 shows the instantaneous $x$-vorticity at $x / d=-0.5$ (upstream edge of the injection hole) and at $x / d=0.5$ (dounstream edge of the injection hole). At $x / d=-0.5$, the beginings of the CVP can clearly be seen. This was also observed in Fig. 3c, and implies that the x-vorticity of the same sign as the CVP is originated on the windward side through the interaction between the jet and the crossflow. Both at $x / d=-0.5$ and 0.5 , the CVP appears to be straddled by an eddy rotating in the opposite direction. This is possibly the horse-shoe vortex that lifts up above the CVP at this low blowing ratio. Reported studies in the literature at higher blowing ratios indicate that the horse-shoe vortex is deflected in the spanwise direction by the jet and travels downstream along the wall parallel to the CVP. In the present case the signature of the horse-shoe vortex in the spanwise direction is not observed, and we believe that at the low blowing ratio considered here, since the jet is immediately bent by the crossflow, the horse-shoe vortex instead of being pushed in the spanwise direction is pushed up in the vertical direction to ride above the CVP.

Figure 9 (a) shows the time-series (presented $2 \Delta t$ apart) of the $x$-vorticity at $x / d=2$. Three distinct structures can be observed: the CVP, the horse-shoe vortex system, and a wall vortex below the CVP. The orientation of the wall vortex and the horse-shoe vortex are in the same direction and opposite to the CVP. The wall vortex is due to the entrainment of the crossflow into the wake of the jet. The entrained crossflow encounters high pressures along the jet-hole centerplane which drives the flow along the wall away from the centerplane, thus establishing a wall-vortex. The horse-shoe vortex is now shifted outwards toward the periodic boundaries, but 
still remains lifted off the surface. In examining the time-series, one notes that the CVP undergoes significant distortion with time. There is also significant spanwise transport of vorticity across the periodic boundaries leading to fairly effective spanuise mixing.

At $x / d=5$ (Fig. 9(b)), a noticeable interaction between the wall-vortex and the CVP is seen. The wall-vortex can be seen to be entrained upwards towards the CVP, and in this process is reoriented into y-vorticity representing wake vortices (seen in Fig. 10 and 11). This reorientation of the wall-vortex system into a wake vortex system has also been reported by Fric and Roshko (1994) and Kelso et al. (1996).

Further dounstream at $x / d=10$ (not shown), we cannot clearly identify the CVP as being an organized structure spanning both sides of the jet-hole vertical midplane. There is significant vortical activity though, but of a much more complex nature, and indicates the persistence of the wake.

Figure 10 shows the instantaneous $\omega_{y}$ contours close to the lower surface at $y / d=0.002$, while Fig. 11 shows the time history of $\omega_{y}$ contours at $y / d=0.0239$ respectively. In Figure 10 , we note that the wake vortices strongly manifest themselves primarily beyond $x / d=5$ which is the approximate location where we noted upward entrainment and reorientation of the wall vortex system (Figure 9(b)). This observation provides further evidence of the correlation between the wall-vortex and the wake-vortex system. The wake vortices are in the form of a series of corotating or counter-rotating adajacent eddies that have features quite distinct from those behind a rigid cylinder. At earlier $x / d$ locations, upstream of $x / d=5$, the wall vortex had remained fairly stable and bound to the near wall-region, and no significant presence of a series of adjacent co- 
and counter- rotating wake vortices was noted. Instead, $y$-vorticity in the near wake $(x / d<5)$ was observed in the form of a near-contiguous elongated chain (see Figure 10 and 11) and stems form the interaction of the jet and the deflected crossflow which leads to significant values of the $\partial w \partial z$ term (and therefore $\omega_{y}$ ) along the spanwise edges of the jet. This vorticity is entrained into the near wake $(x / d<5)$, and eventually breaks down further downstream, contributing also to the strong wake vortex system observed beyond $x / d$ of 5 . The time series behavior shown in Fig. 11 also indicates that the wake structure oscillates around the jet-hole center-plane in the near field, but exhibits significant asymmetry in the far field. At time instances $t_{1}, t_{3}$ and $t_{5}$, for example, the wake vortex system beyond $x / d$ of 6 is skewed considerably towards positive $z / w$ values. This departure from symmetry was also noted earlier in velocity vector plots of Fig. 5c.

Figure 12 presents the instantaneous pressure contours at $y / d=0.0239$ at four timeinstances. The adverse pressure gradient upstream of the jet can clearly be seen, and is responsible for the horse-shoe vortex system. In the jet-hole region, and downstream of it, the pressure distribution shows a necklace pattern. Note that in the region $-0.5<\mathrm{x} / \mathrm{d}<0.5$ there is a strong pressure gradient in the crossflow directed inwards in the spanwise direction toward the jet. This inward pressure gradient combined with the adverse pressure gradient the flow encounters at the jet interface leads to the generation of $x$-vorticity of the same-sign as the CVP along the spanwise boundaries of the jet-hole $(z / w= \pm 0.5)$. This mechanism therefore appears to be responsible for the initial development of the CVP.

Downstream of the jet-hole, the spanwise pressure gradient is directed inwards towards the jet centerline. This pressure gradient is responsible for the inward entrainment of the 
crossflow that is the source of the wall vortex system. Note that downstream of about $x / d=5$, the coherence of the pressure contours is somewhat lost leading to the asymmetric wake pattern reported earlier.

\section{Time-averaged Statistics}

Figure 13 and 14 show the ms contours at $x / d=0.5,2,5$ and 10 . At $x / d=0.5, v_{r m s}$ and $w_{r m s}$ are comparable in magnitude with the latter attaining slightly larger values, but $u_{\mathrm{rms}}$ is nearly twice the $v_{r m s}$ and $w_{r m s}$ values. The $u_{m s}$ and $w_{r m s}$ plots correlate well with each other exhibiting maximas in regions of high unsteady $\omega_{x}$ vorticity, that is in the outer regions of the CVP and the CVPhorseshoe vortex interface (see Fig. 8) that exhibit a significant level of unsteadiness, and near the spanwise periodic boundaries across which significant vorticity transport occurs (Fig. 8 shows only one instance in time, and shows high vorticity near the $z / w=-3$ boundary. At other instances in time high vorticity is seen at the $z / w=3$ boundary as shown in Fig. 9). The $v_{m s}$ and $t_{r m s}$ profiles correlate well with each other, but do not show a strong correlation with the $u_{\mathrm{rms}}$ and $w_{\mathrm{mms}}$ plots. In fact the peak values are obtained near $y / d=1$, and appears to correspond to the extent of the vertical jet penetration. The interface between the jet and the crossflow, is characterized by Tollmein-Schlichting instabilities and high spanwise vorticity $\omega_{z}$ (see Fig. 7), and is therefore associated with high $v_{m s}$. Since the jet and the crossflow are at different temperatures, their interface is also associated with high $t_{\mathrm{ms}}$. The aforedescribed correlation between the rms values and the coherent structures imply that the large scales are largely responsible for the production of turbulent stresses. At $x / d=2$, the behavior of the rms profiles is quite similar to that at $x / d=0.5$, except that the $t_{\mathrm{m} m}$ profiles shows high values both in regions of high $v_{\mathrm{mms}}$ (interface between the 
upper edge of the jet and the crossflow) and high $u_{\text {rms }}$ (interface between the CVP and the crossflow). Further downstream $\left(x^{\prime} \mathrm{d}=5\right.$ and 10$)$ the jet deflection is complete. and the CVP dominates growing in size and strength. All the rms-profiles now correlate with the high vorticity regions of the CVP, and as a consequence correlate well with each other. At $x / d=10$, we still observe $u_{\mathrm{rms}}>v_{\mathrm{ms}}>w_{\mathrm{mms}}$. although the relative magnitudes are closer to each other than they were at $x / d=0.5$. Note that the time-averaged rms-profiles at $x / d=10$ show reasonable symmetry around $z / x=0$, while the instantaneous profiles shown earlier (Fig. 5c and 8-11) displayed significant asymmetry in the spanwise direction.

The ms profiles in the $z / w=0$ plane are shown in Figure 15. All the peak values occur downstream of the jet injection $(x / d=0)$, but the upwash effect is clearly evident. High rms values enter the computational domain from the upstream boundary, and the flow development downstream of the injection point is constrained by these high rms values. The $u_{r m s}$ value peaks first in the $x$-direction (near $x / d=5$ ) and occurs closest to the wall $\left(y / d=0.25\right.$ ), while the $v_{m s}$ peaks around $x / d=6.5$. These peak locations correspond with the location where the shear layer vortices saturate in strength (see Fig.7). The other $\mathrm{mms}$ values $w_{\mathrm{ms}}$ and $t_{\mathrm{ms}}$ peak at an $\mathrm{x} / \mathrm{d}$ in the vicinity of 7. Note that in the vicinity of this $x / d$ (in the range of 5-7), significant reorientation of the wall vortex system into a wake vortex system occurs, and therefore the wake- and wall-vortex structures structures apparently play an important role in the $w_{m s}$ and $t_{m s}$ values.

Figure 16 and 17 present the Reynolds stress components at $x / d=0.5,2,5$ and 10 . As expected, $u^{\prime} v^{\prime}$ is observed to be symmetric about $z / w=0$, and $u^{\prime} w^{\prime}$ and $v^{\prime} w^{\prime}$ are observed to be antisymmetric about $z / w=0$. The high $u^{\prime} v^{\prime}$ values are associated with the jet-crossflow interface 
shown in Fig. 7, where shear layer vortices primarily form. At $\mathrm{x} / \mathrm{d}=10$, distinct regions of upper and lower interfaces are no longer clearly evident and a large patch of high $u^{\prime} v^{\prime}$ is noted in the jetflow region. High values of $u^{\prime} v^{\prime}$ are also associated with the vortical structures transported across the periodic boundaries. These structures lead to large values of $\partial u / \partial y$ and therefore $u^{\prime} v^{\prime}$. The $u^{\prime} w^{\prime}$ and $v^{\prime} w^{\prime}$ values are high in regions of large $\partial u / \partial z$ and $\partial v / \partial z$ respectively. These regions are again associated with the jet-crossflow interfaces and the vortical structures crossing the periodic boundaries. Note that the clover-leaf shape and the sign of the $v^{\prime} w^{\prime}$ contours near the wall for $x / d>5$ indicate the existence of counter-rotating eddies near the wall, i.e., the CVP and the wall vortex, in the $y-z$ plane. In comparing the magnitudes of the shear stresses, in the near field $(x / d<2), u^{\prime} w^{\prime}>u^{\prime} v^{\prime}>v^{\prime} w^{\prime}$ with $u^{\prime} w^{\prime}$ being substantially higher presumably due to large $\partial u / \partial z$ values near the edges of the jet. Further downstream, $u^{\prime} w^{\prime}$ and $u^{\prime} v^{\prime}$ become more comparable in magitude.

Figure 18 presents the Reynolds stresses in the $z / w=0$ plane. The peak shear stresses, in all cases occur downstream of $x / d=5$. The $u^{\prime} v^{\prime}$ contours correlate best with the $v_{\text {mms }}$ contours (Fig.15) and therefore correlate with the shear layer vortices, and peak at the locations where these vortices saturate in strength. At $x / d=5$, for example, the peak $u^{\prime} v^{\prime}$ value shown in Fig. 17 is 2.85 and its $y / d$ location correlates with the shear layer vortices, while secondary bimodal peaks (of the order of 1) occur at $y / d=0.25$ which correlates with the CVP and the horseshoe vortices. The $v^{\prime} w^{\prime}$ contours appear to be consistent with the nature of the near-wall flow structure in the $y-z$ plane. Close to the wall, the wall vortex produces negative $v^{\prime} w^{\prime}$ values. The wall vortex 
system gains prominence, as seen earlier. around $x / d=5$ and therefore large negative $v^{\prime} w^{\prime}$ values are obtained in the vicinity of this $x / d$ value. Further downstream, the wall vortex is entrained as an upflow into the CVP. and as seen in Fig. 11 , wake vortices are noted. The $v^{\prime} w^{\prime}$ contours in this region show a more complex behavior and are positive. The $u^{\prime} w^{\prime}$ contours also correlate well with the crossflow entrainment. They peak where the crossflow is a maximum (around $x / d=5$ ), and decay further downstream.

Figure 19 and 20 present the turbulent heat flux correlations at $x / d=0.5,2,5$ and 10 . The turbulent heat flux correlations appear to correlate reasonably well with the rms values and the behavior of the large scale vortical structures, thus indicating the important role these structures play in the turbulent heat transport. In comparing the magnitudes $u^{\prime} t^{\prime}>w^{\prime} t^{\prime}>v^{\prime} t^{\prime}$ in the near field $(x / d<5)$ indicating that the streamwise velocity fluctuations are most responsible for the heat transfer from the surface. Further downstream, at $x / d=10$, the $v^{\prime} t$ ' values become comparable in magnitude to the $u^{\prime} t^{\prime}$ values, while the $w^{\prime} t$ ' dccrease in magnitude and become less important. The heat flux distributions at $z / d=0$ in the $x-y$ plane are shown in Fig. 21, and support the above observations. The maximum $w^{\prime} t^{\prime}$ occurs near $x / d=5$ where there is significant spanwise entrainment of the crossflow. The peak in the $u^{\prime} t^{\prime}$ and $v^{\prime} t^{\prime}$ occur downstream of $x / d=5$ and at a $y / d$ location of approximately 0.5 . These peak values are therefore associated with the spanwise shear layer vortical structures (Fig.7).

Figure 22 presents the turbulent viscosity factor $v_{t, i j}$ calculated as: 


$$
v_{t .1 j}=\frac{\overline{-u_{i} u_{j}}}{\frac{\partial \overline{u_{i}}+\frac{\partial \overline{u_{j}}}{\partial x_{j}}+\frac{\partial x_{i}}{j}}{\partial}}
$$

where all terms on the right hand side of the above equation are computed from the DNS results. The viscosity factors are presented in a $x-z$ plane corresponding to a $y / d=0.1085$. While all three viscosity factors $v_{t, 12}, v_{t, 13}, v_{t .23}$ were computed, only $v_{\mathrm{L}, 12}$ is presented. Note that the magnitudes of the individual viscosity factors $v_{t, 12}, v_{t, 13}, v_{t, 23}$ at the same point are substantially different in magnitude indicating the anisotropy in the flowfield. The $v_{t, 12}$ clearly corresponds to the wake structure shown in Fig. 11, and indicates that the large-scales clearly have a role in the values of the turbulent viscosity. The footprint of the horseshoe can also be

seen upstream of the jet hole. The $v_{t, 13}, v_{t, 23}$ values show less coherence with respect to the large scale structures, and are characterized more by patches of high positive and negative viscosity factors. The positive and negative values of the viscosity factors are associated with the fact that both the turbulent shear stress and the mean strain rate can have both positive and negative values. Clearly, any use of an isotropic turbulent viscosity is going to be unsuccessful in capturing the true behavior.

\section{Concluding Remarks}

A Direct Numerical Simulation of a film coolant jet injected normally into a crossflow has been presented. The crossflow Reynolds number is 5600 and the blowing ratio is 0.25 . Both flow and heat transfer predictions are presented. The following major observations are made:

1. Jet penetration is limited to 1.8 hole diameters, with the primary crossflow velocity relatively unaffected beyong 1 hole diameter. 
2. A weak horse-shoe vortex system consisting of two clockwise eddies is noted within one hole diameter upstream of the jet. This horseshoe vortex is deflected upwards and rides over the CVP in the near field of the jet.

3. The near-field past jet reattachment is characterized by developing boundary layer behavior with ejection and sweep events commonly termed as turbulent bursts. However, these events are largely controlled by the large scale vortical structures.

4. The origins of the CVP is associated with the $\mathrm{x}$-vorticity generated by the interaction of the injected jet with the crossflow. Therefore hole-boundary-layer vorticity is not essential for the generation of the CVP.

5. There is a significant amount of crossflow in the near field $(x / d<5)$ of the jet. This crossflow encounters an adverse gradient along the jet-hole centerline and is responsible for the formation of the wall vortex system. The wall vortex is fairly stable for $x / d$ up to 5 , but beyond that is entrained into the CVP and is reoriented as a wake vortex system.

6. Along the upper edges of the jet, Tollmein-Schlichting instability leads to the formation of the shear layer vortices. These vortices are peeled and convected downstream.

7. The $v_{m s}$ and $t_{m s}$ contours appear to be most strongly correlated with each other and with the shear layer vortices in the near field. The $u_{m s}$ and $w_{m s}$ contours appear to be the larges tat the interface between the CVP and the horse-shoe vortex and along the periodic boundaries at the locations associated with significant spanwise crossflow. In general, $u_{m s}>w_{r m s}>v_{r m s}$. The strong correlation of these statistics with the large scale structures point to the importance of the latter in controlling the surface heat transfer and the near field mixing between the crossflow and the coolant stream. 
8. The viscosity factors show considerably anisotropy and in the horizontal (x-z) plan show correlation with the large scale structures. Existing statistical models based on isotropy are clearly going to be inadequate in modeling this complex flow. Current efforts are focussed in examining the budgets of the turbulence kinetic energy and the dissipation rates, in order to provide some guidance toward turbulence modeling.

\section{Acknowledgements}

This work was supported by an AST grant from the Turbomachinery Fluid-Physics group of NASA-Lewis with Dr. Chi Wang and Dr. Ray Gaugler serving as monitors. This support is gratefully acknowledged. We also appreciate the help of Professor Joel Tohline of the Physics Department at LSU for providing us with computer time to run on the MasPar computing system.

\section{References}

Andreopoulos, J., 1985, On the structure of jets in a crossflow, Journal of Fluid Mechanics, Vol. 157

Chorin, A. J., 1967, A numerical method for solving incompressible viscous flow problems. Journal of Computational Physics, 2:12-26

Demuren, A. O., 1993, Characterstics of three dimensional jets in crossflow, International Journal of Engineering Science, No. 6, pp. 899-913

Fric, T. F., and Roshko, A., 1994, Vortical structure in the wake of a transverse jet, Journal of Fluid Mechanics, Vol. 279, pp. $1-47$

Foss, J., 1980, Interaction region phenomenon for the jets in a cross-flow problem, Rep. SFB 80/E/161, Univ. Karlsruhe

Gavrilakis, S., 1992, Numerical simulation of low reynolds numner turbulent flow through a straight square duct, Journal of Fluid Mechanics, Vol. 244, pp. 101-129

Huser, A., and Biringen, S., 1993, Direct numerical simulation of turbulent flow in a square duct, Journal of Fluid Mechanics, Vol. 257, pp. 65-95

Jones, W. P., and Willie, M., 1996, Large eddy simulation of a round jet in crossflow, Engineering Turbulence Modeling and Experiments 3, Ed. Rodi, W. and Bergeles, G., pp. 199-209 
Kasagi. N., Tomita, Y.. and Kuroda, A., 1992, Direct Numerical Simulation of Passive Scalar Field in a Turbulent Channel Flow, Transactions of the ASME, Vol. 114, pp. 598-606.

Kelso. R. M.. Lim. T. T.. and Perry A. E., 1996 An experimental study of jets in crossflow. Journal of Fluid . Mechanics, Vol. 306, pp. 111-144

Kim, S. W., and Benson. T. J., 1992, Calculation of a circular jet in crossflow with a multiple time scale turbulence model, International Journal of Computational Physics, Vol. 59, pp. 308

Kim, J., Moin P., and Moser, P., 1987, Turbulence statistics in fully developed channel flow at low reynolds number, Journal of Fluid Mechanics, Vol. 177, pp. 133-166

Kim, J., and Moin, P., 1989, Transport of passive scalars in a turbulent channel flow, in Turbulent Shear Flow's V1, J. C. Andre et al eds., Springer Verlag, Berlin, pp. 85-96

Kreplin, H., and Eckelman, H., 1979, Behavior of the three fluctuating velocity components in the wall region of a turbulent channel flow, Phys. of Fluids, Vol. 22, pp. 1233-1239

Lu, D. M., and Hetsroni, G., 1995, Direct numerical simulation of a turbulent open channel flow with passive heat transfer, Int. J. Heat and Mass Transfer, Vol. 38, pp. 3241-3251

Lyons, S. L., Hanratty, T. J., and McLaughlin, J. B., 1991, Direct numerical simulation of a passive heat transfer in a turbulent channel flow, Int. J. Heat Mass Transfer, Vol. 34, pp. 1149-1161

Moser, R. D., and Moin. P., 1984, Direct numerical simulation of curved turbulent channel flow, NASA TM 85974

Muldoon, F., and Acharya, S., 1998, Dynamics of large-scale structures for jets in crossflow, 43rd Intl. Gas Turbine Institute Conference, Stockholm, Sweden; also NASA/CR-1998206606

Najjar, F., M., and Vanka, S. P., 1994, Simulations of unsteady fluid flows on the CM-5. Symposium on Advances in Computational Methods in Fluid Dynamics, ASME/Fluid Division Summer Meeting.

Orszag, S. A., and Kells, S. C., 1980, Transition to turbulence in plain Poiseuille and plane Couette flow, Journal of Fluid Mechanics, Vol. 96, pp. 159-205

Orszag. S. A., and Patera, A. T., 1983, Transition to turbulence in plane Pouiseuille and plane Couette flow, Journal of Fluid Mechanics, Vol. 128, pp. 347-385

Pointel, G., Acharya, S., and Sharma, C., "DIrect Numerical SImulations of Convective Heat Transfer", 1996 Energy Technology Conference and Exposition, Houston, January 1996.

Patankar, S. V., Basu, D. K., and Alpay, S. A., 1977, Prediction of the three dimensional velocity field of a deflected turbulent jet, Transactions of the ASME

Rai, M., and Moin, P., 1989, Direct simulations of turbulent flow using finite-difference schemes. ALAA Paper 89-6039. 
Simutani, Y., and Kasagi, N., 1995. Direct numerical simulation of turbulent transport with uniform wall injection and suction. Al.AA Journal, Vol. 33. pp. 1220-1228

Sykes. R. I.. Lewellen, W. S., and Parker, S. F., 1986. On the vorticity dynamics of a turbulent jet in a crossflow, Journal of Fluid. Mechanics, Vol. 80. pp. 49-80

Yuan. L. L.. and Street. R. L., 1996, Large eddy simulation of a jet in crossflow. AS.ME Fluids Engineering Division, Vol. 242 

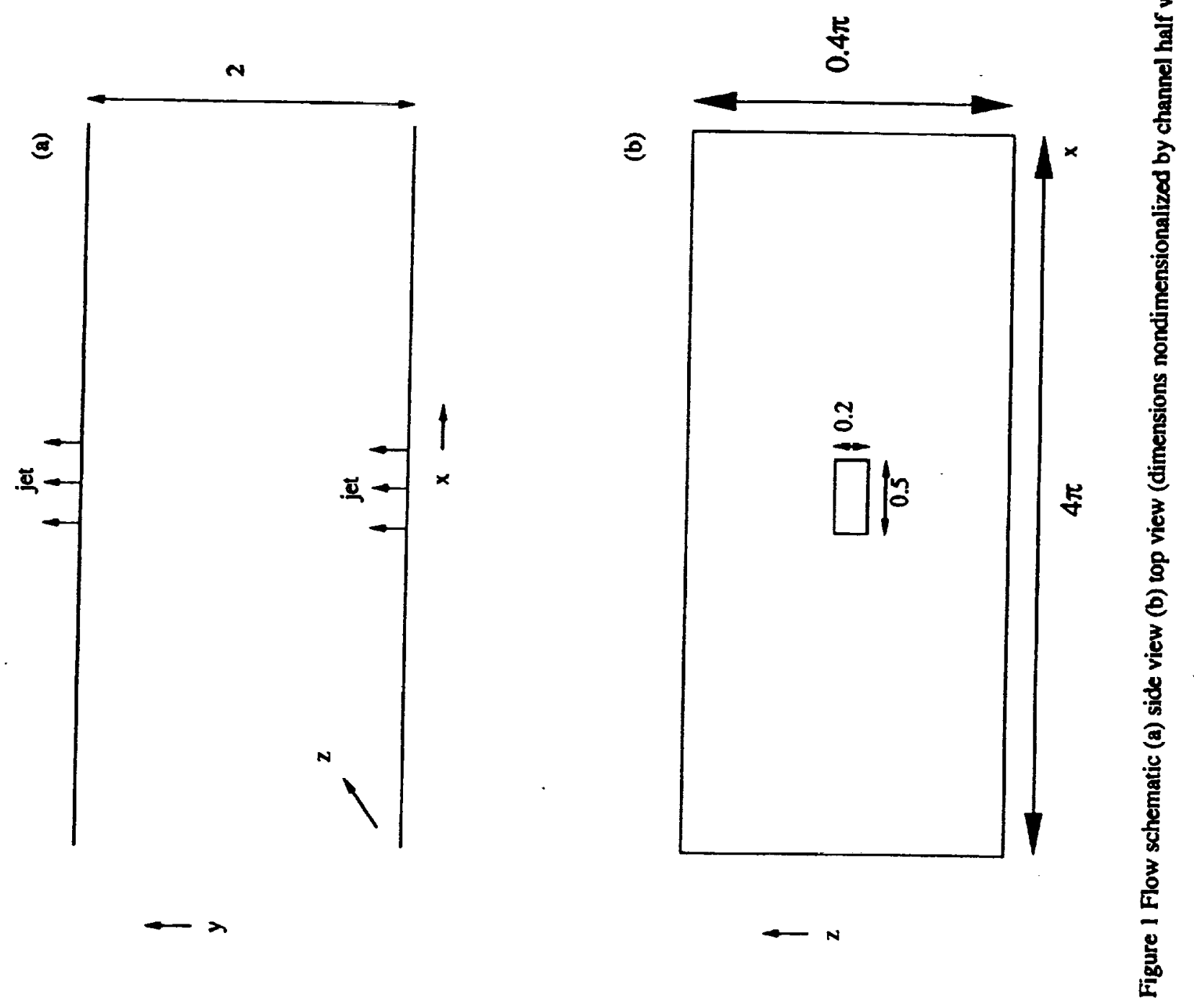

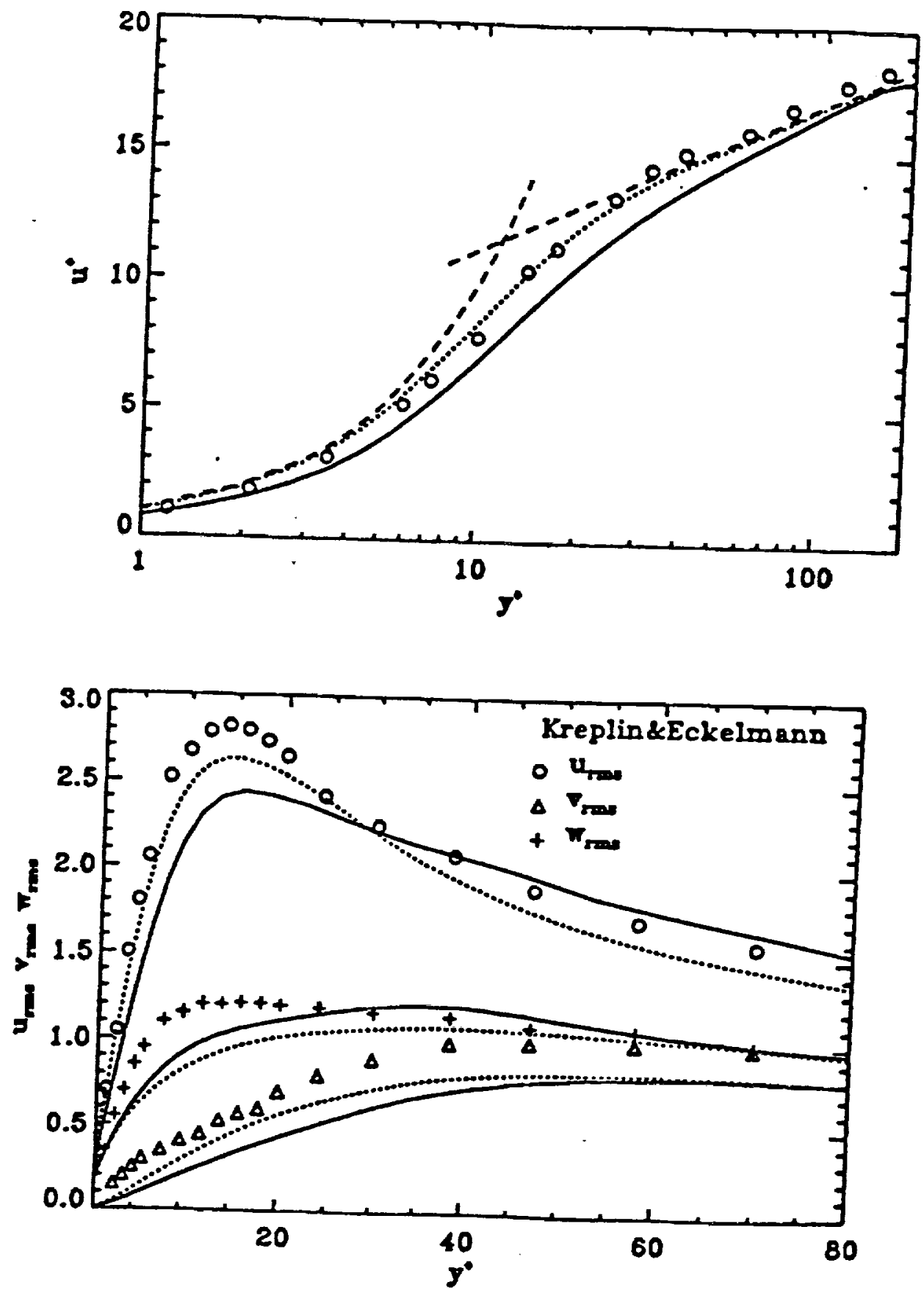

Figure 2: Comparison of DNS predictions of Pointel et al., $1996(\longrightarrow)$ with the DNS predictions of Kim et al., 1987 (-) and experimental data of Kreplin and Eckelman $(0,+, \Delta)$. Law of the wall shown by (- . . - -) lines 


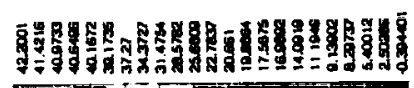

ฮ

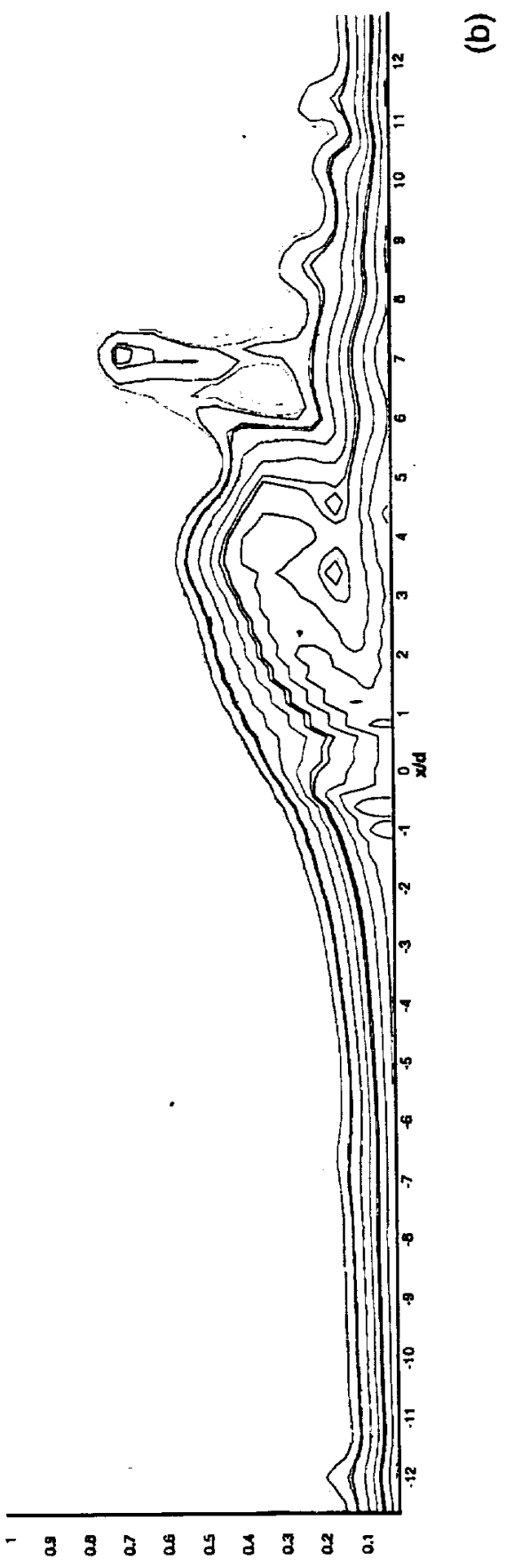

$\mathrm{p} \mu$

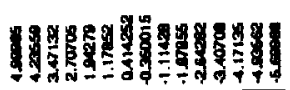

IIIT
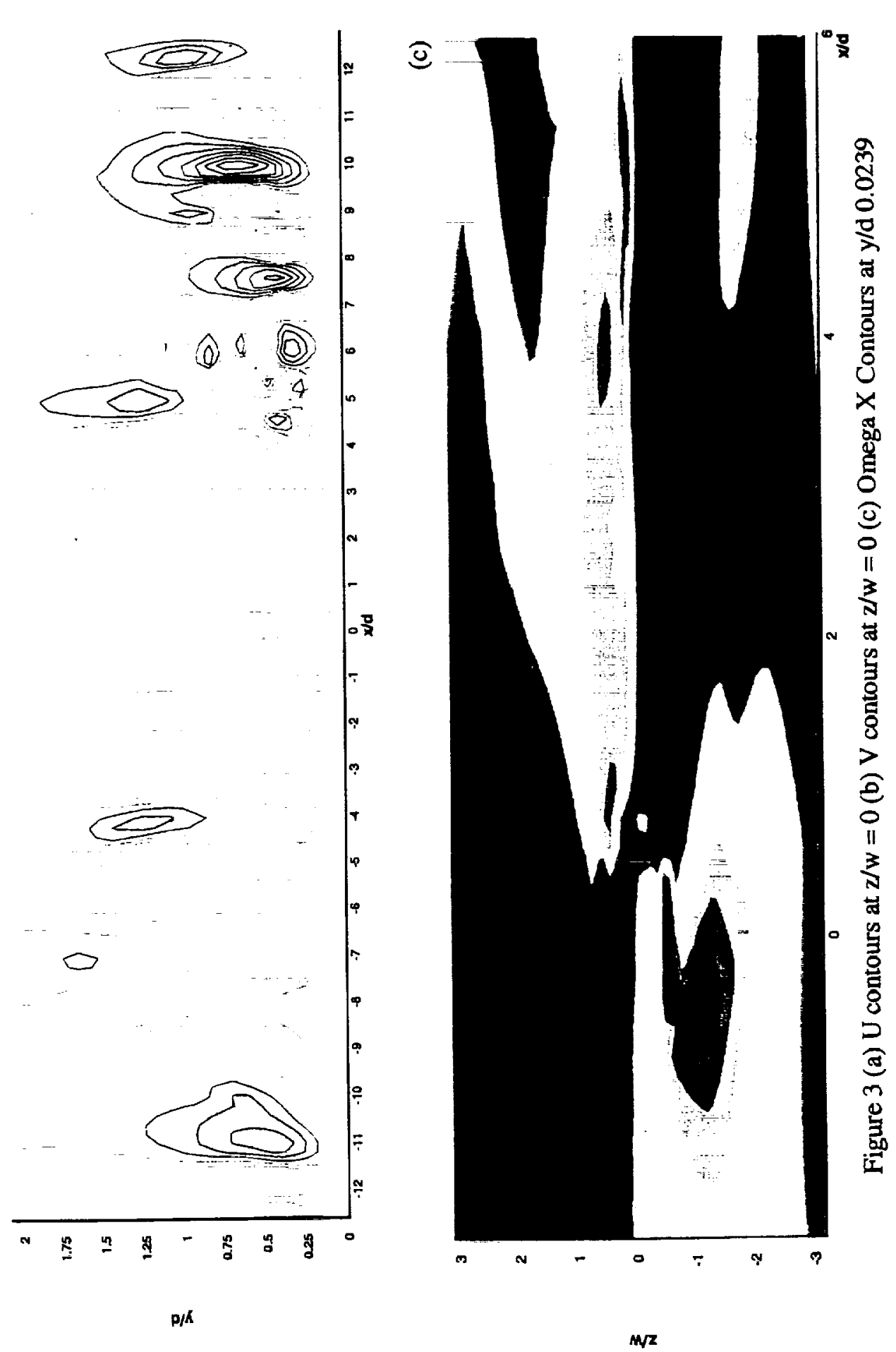

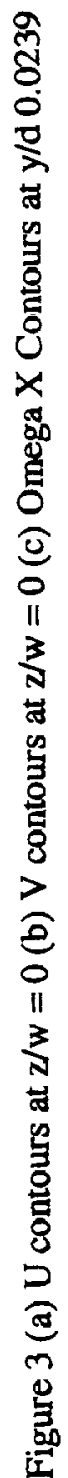

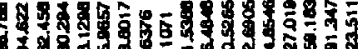

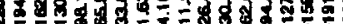



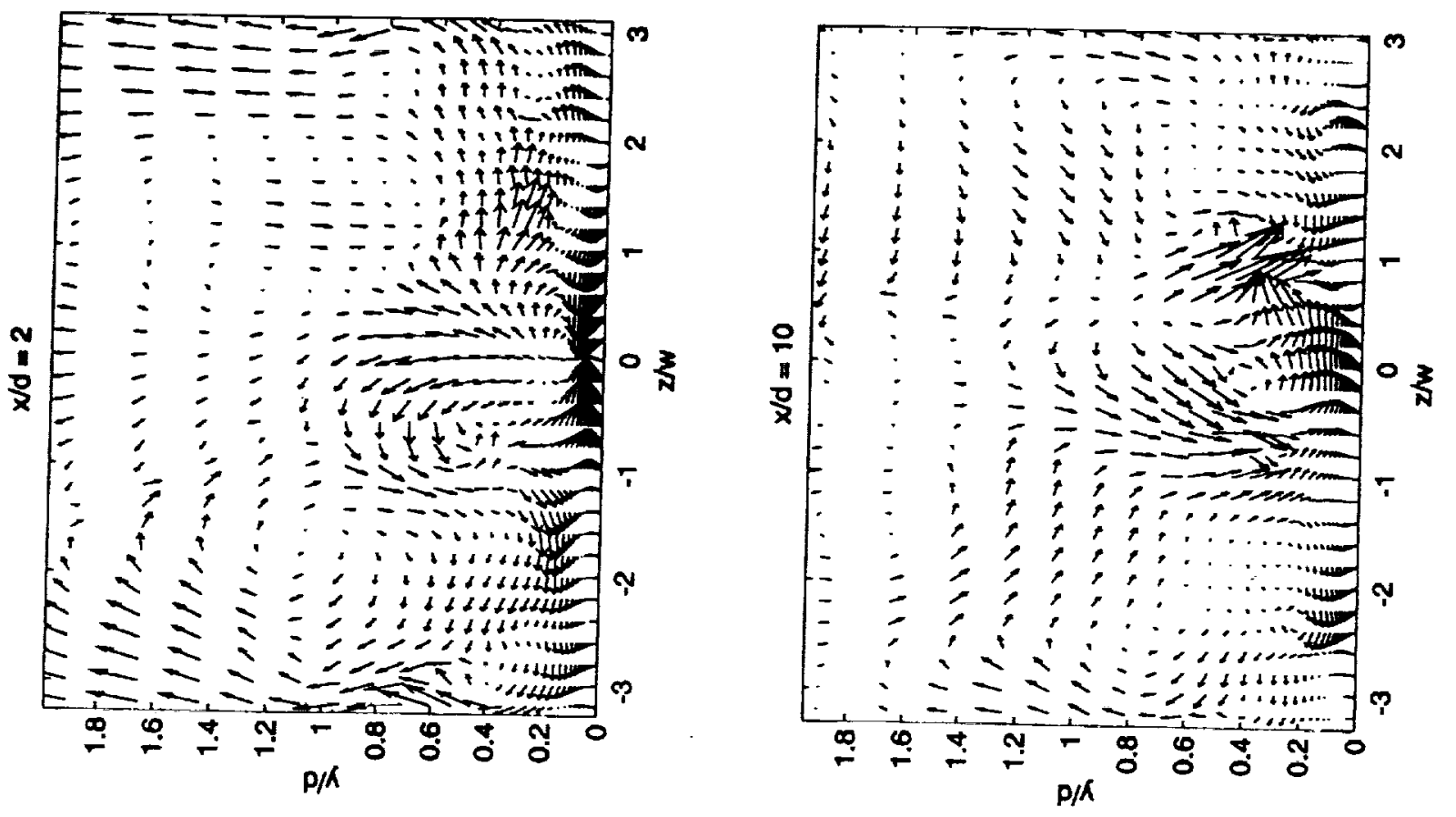

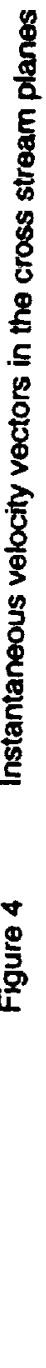
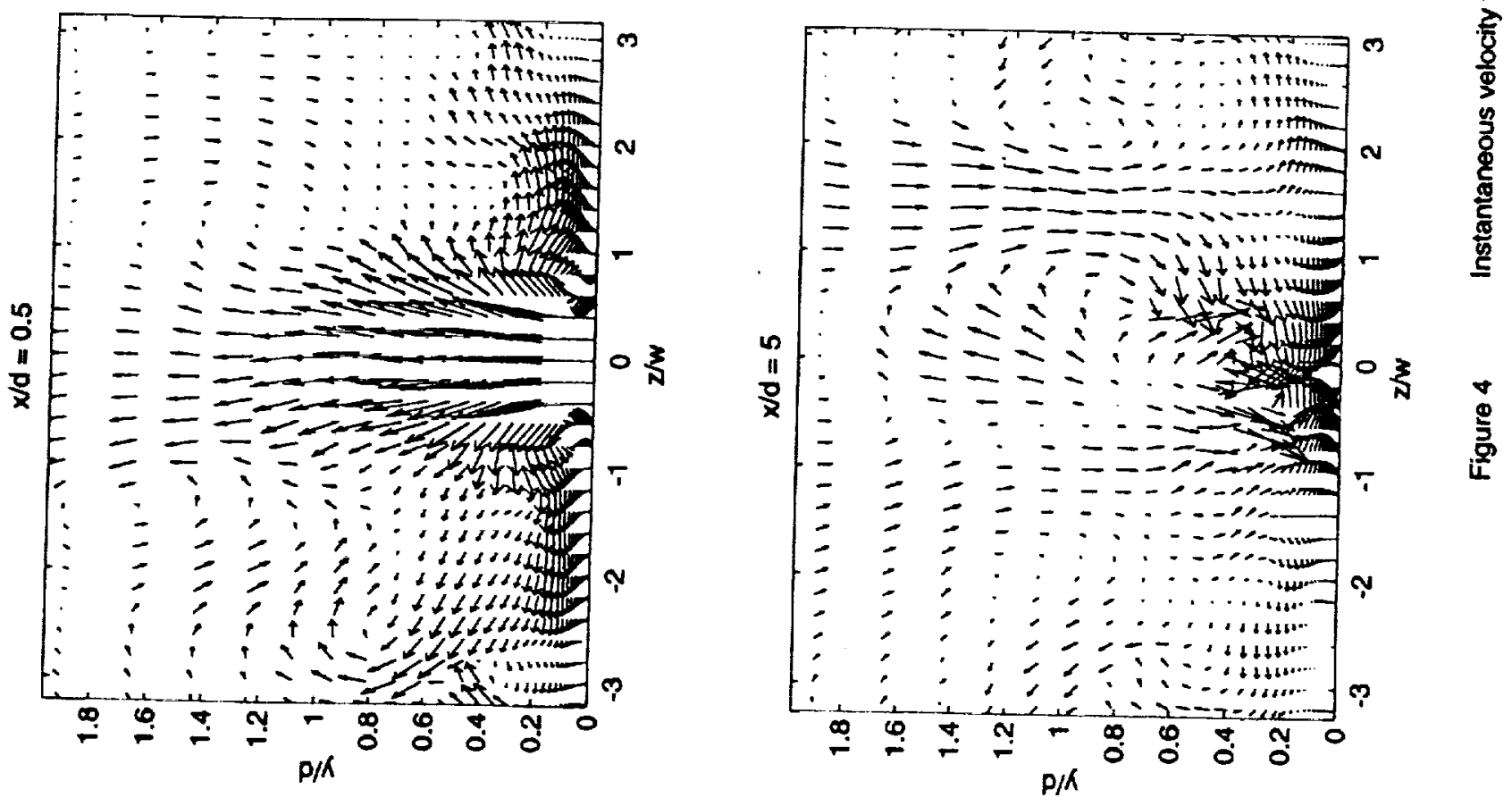

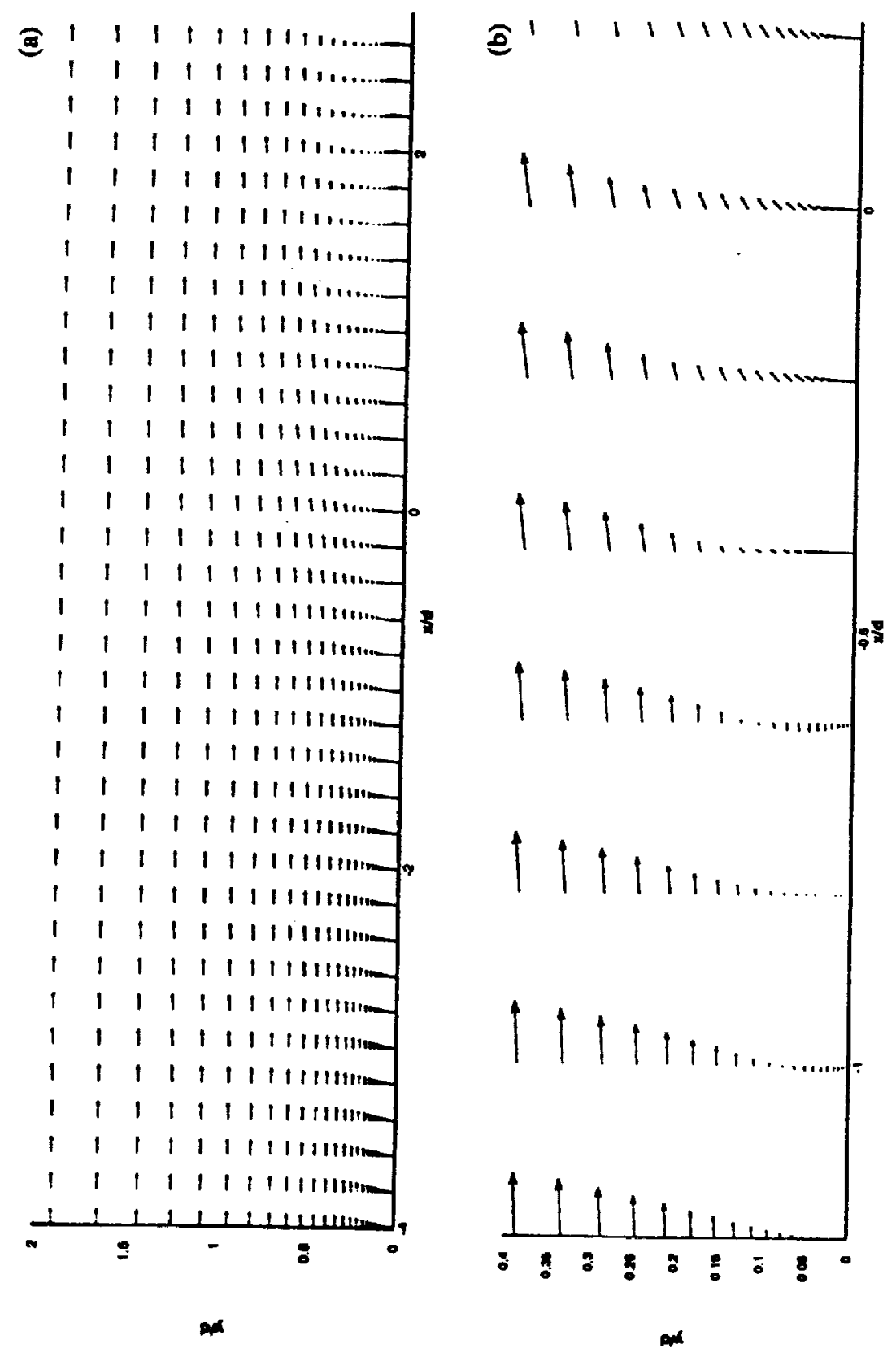

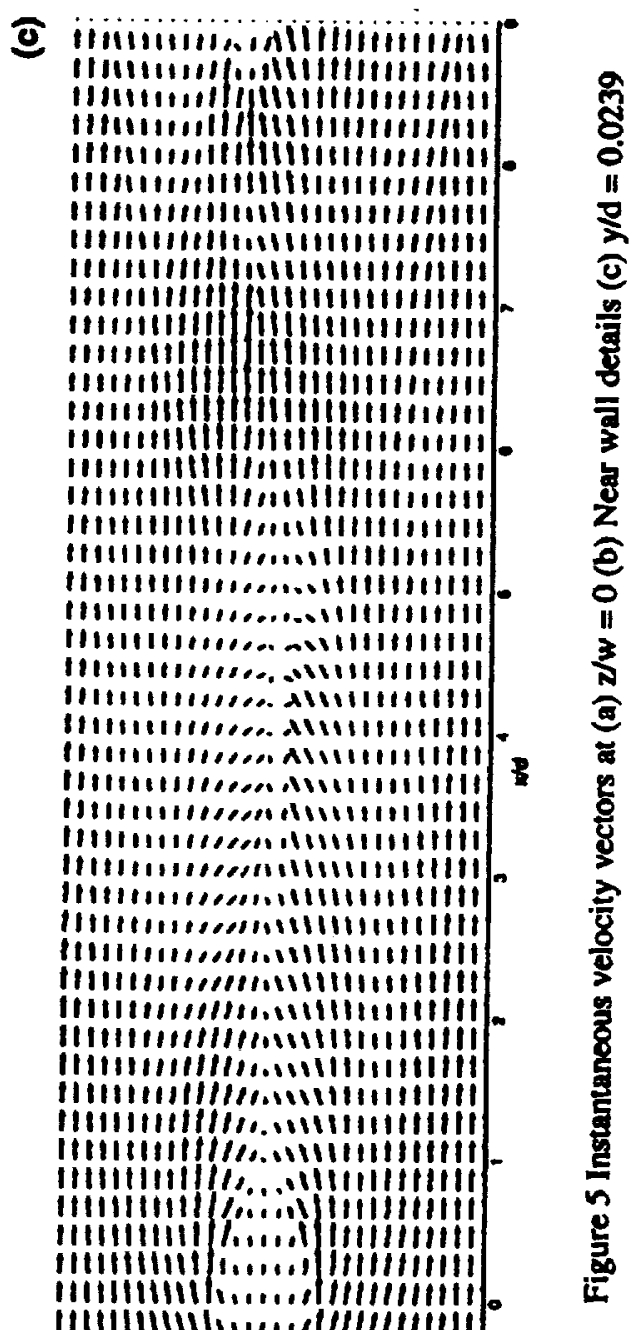

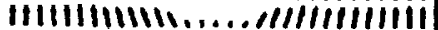
IIIIIIIIII,

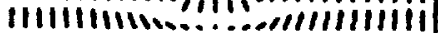
IIIIIIIII).

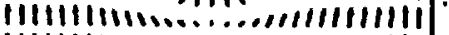
IIIIIIIIIII.........entilitiliti

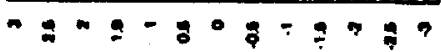



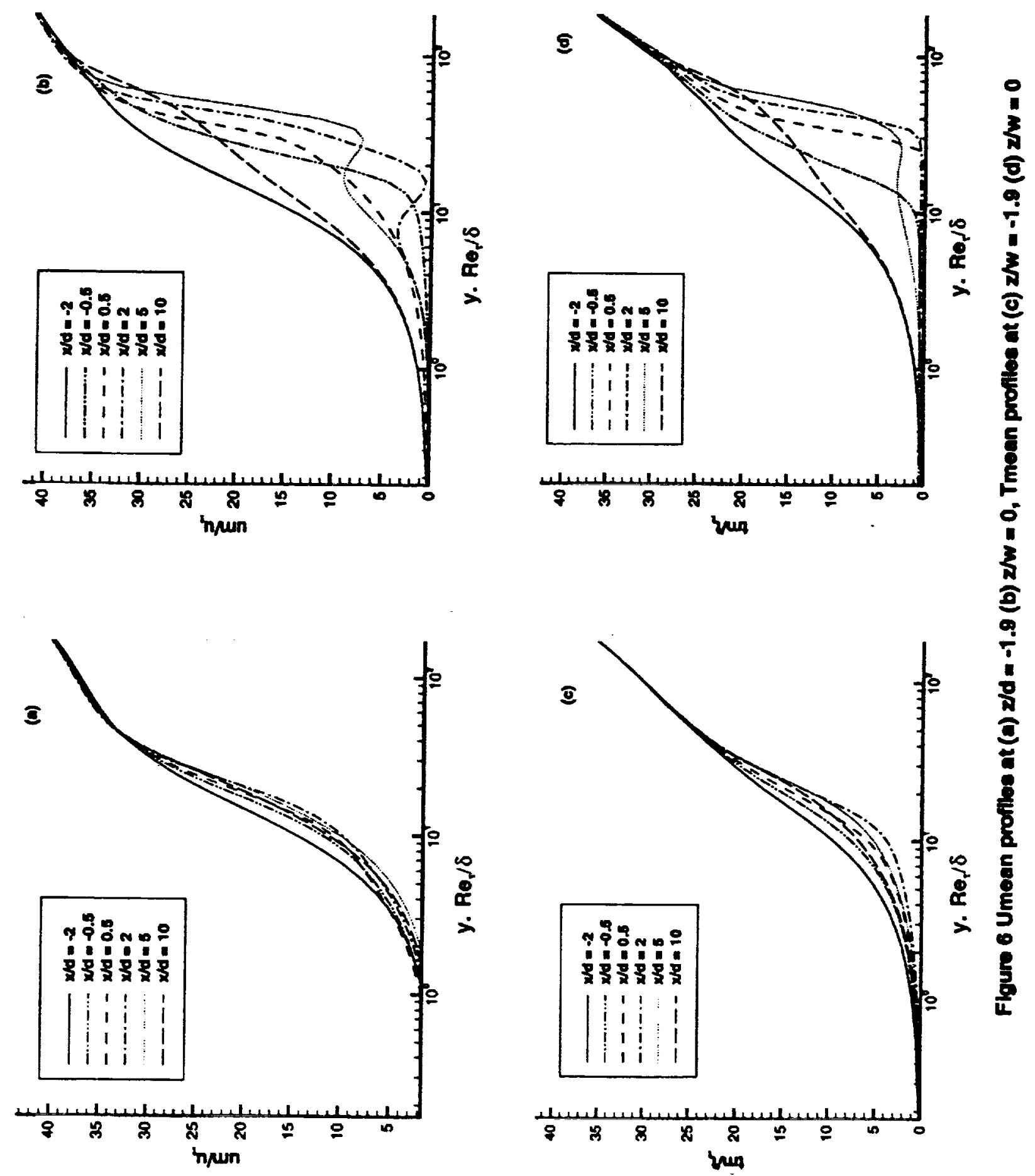

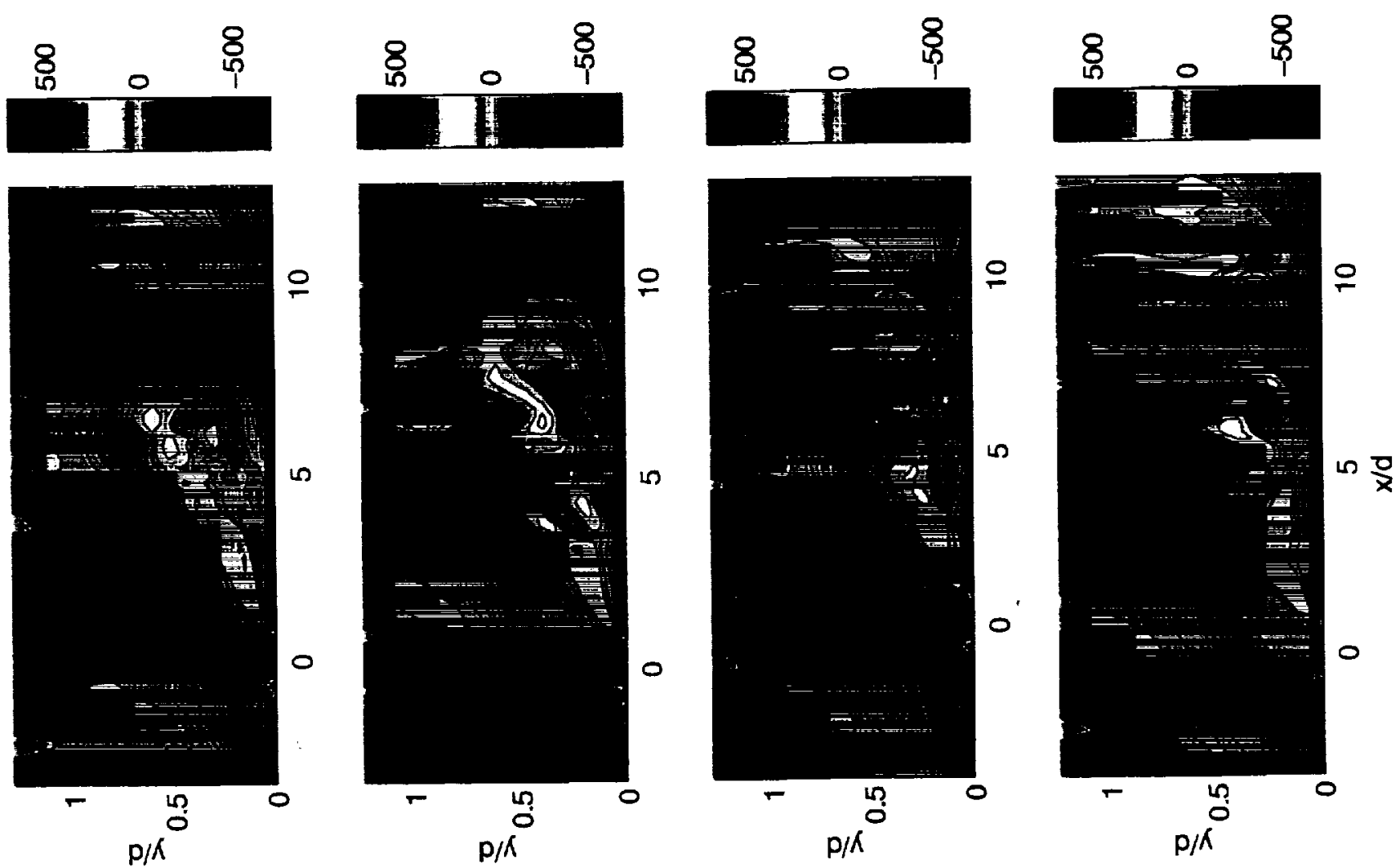

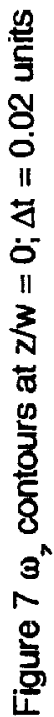
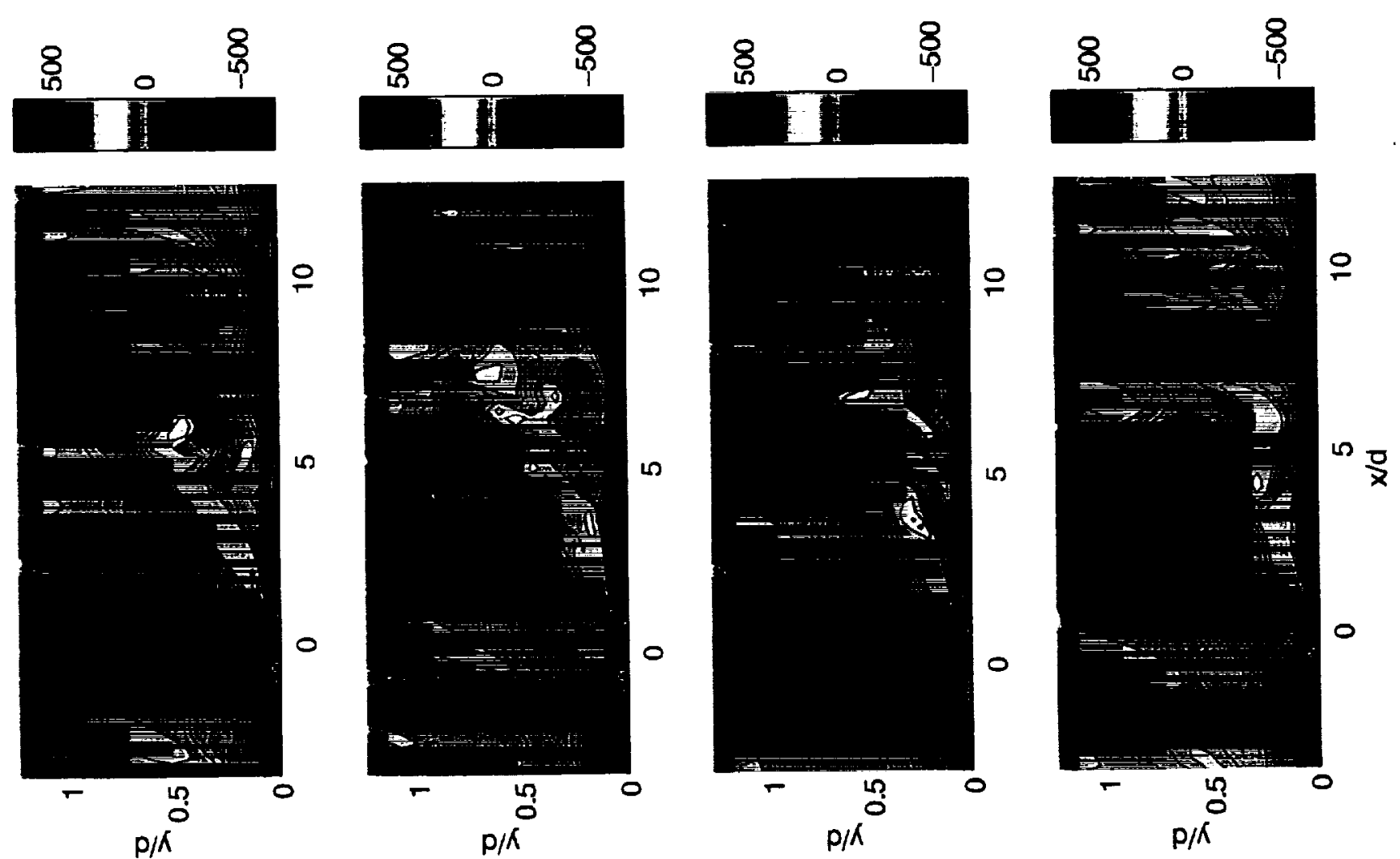


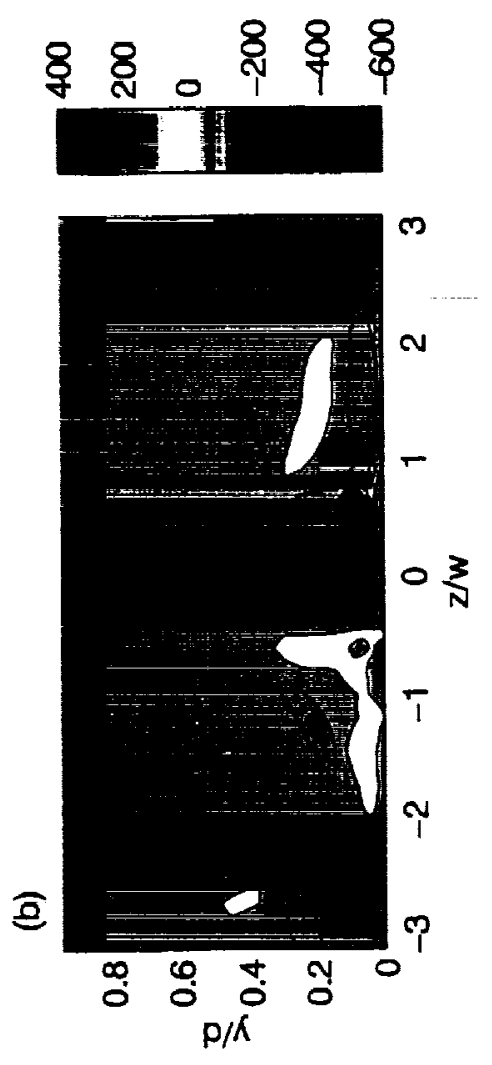

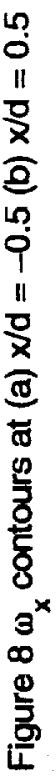

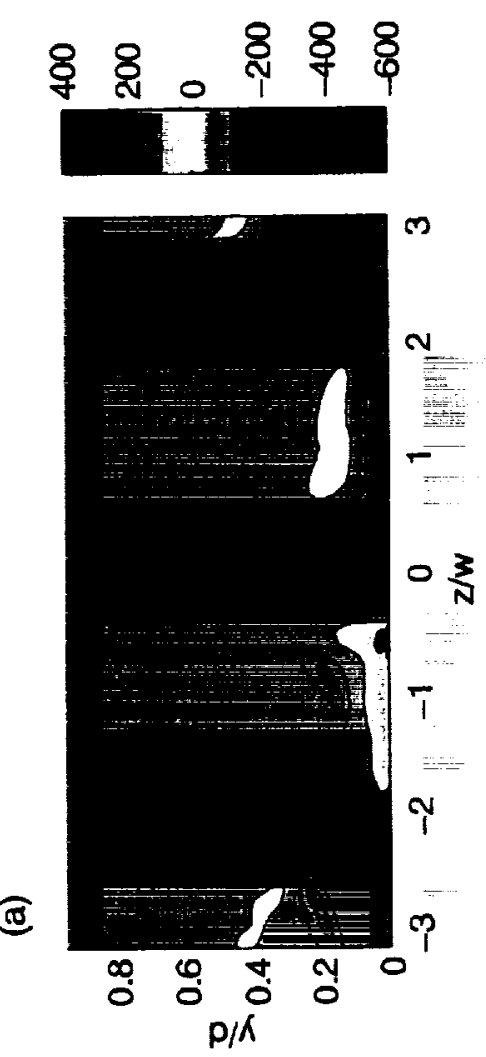


8 8 8 \& 8 \% 8
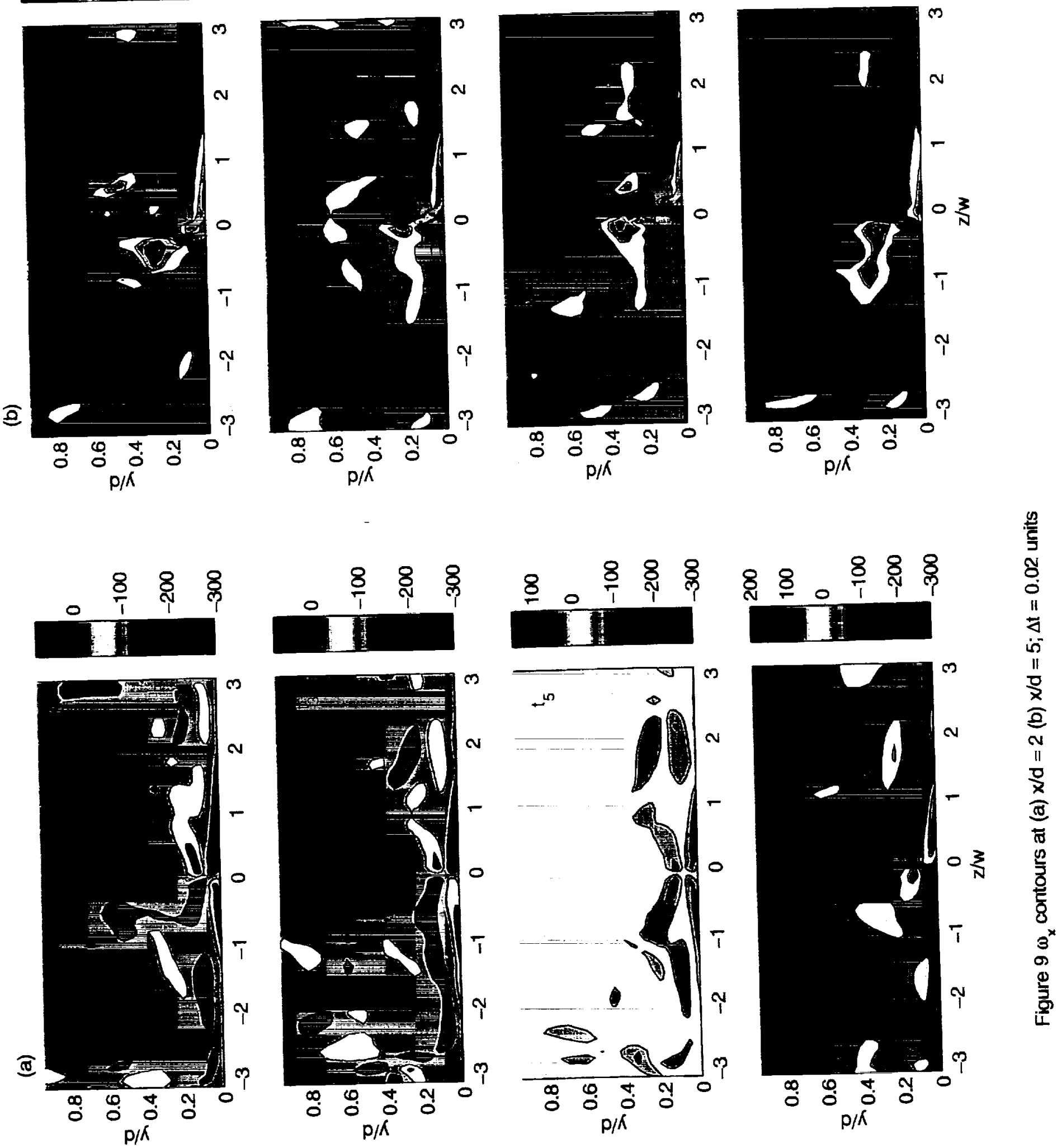

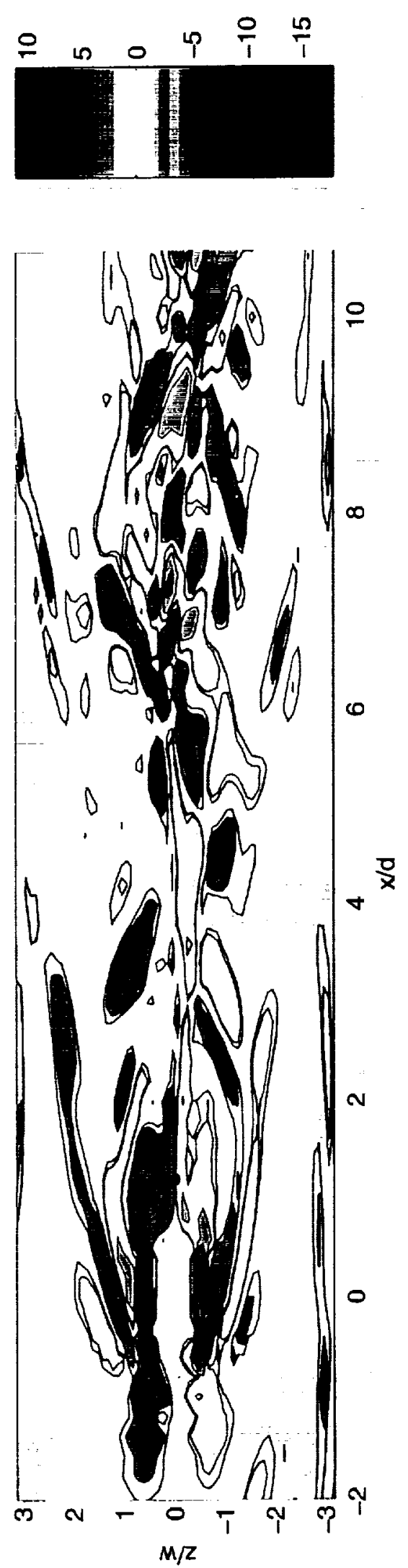

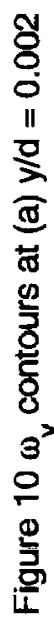



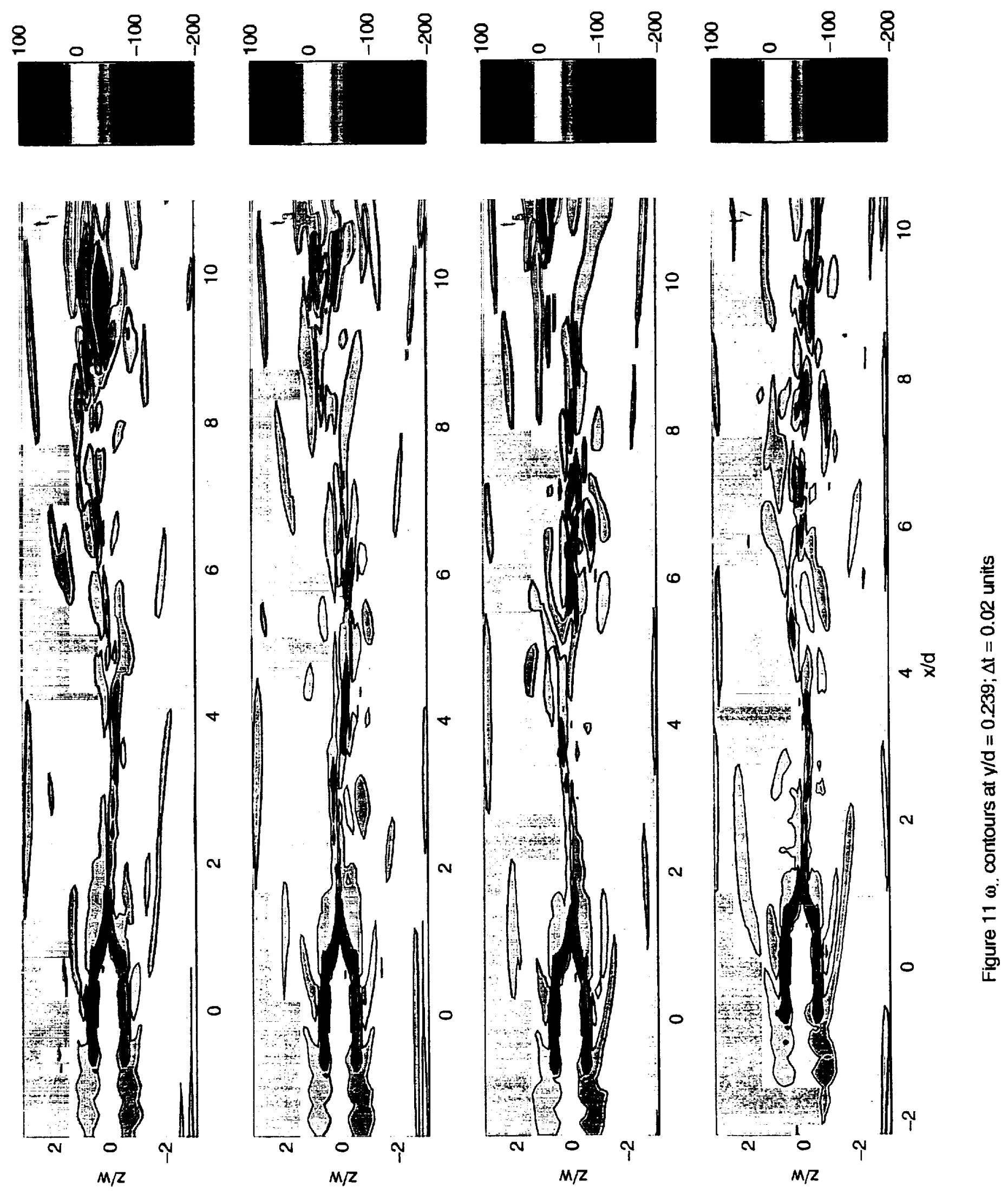

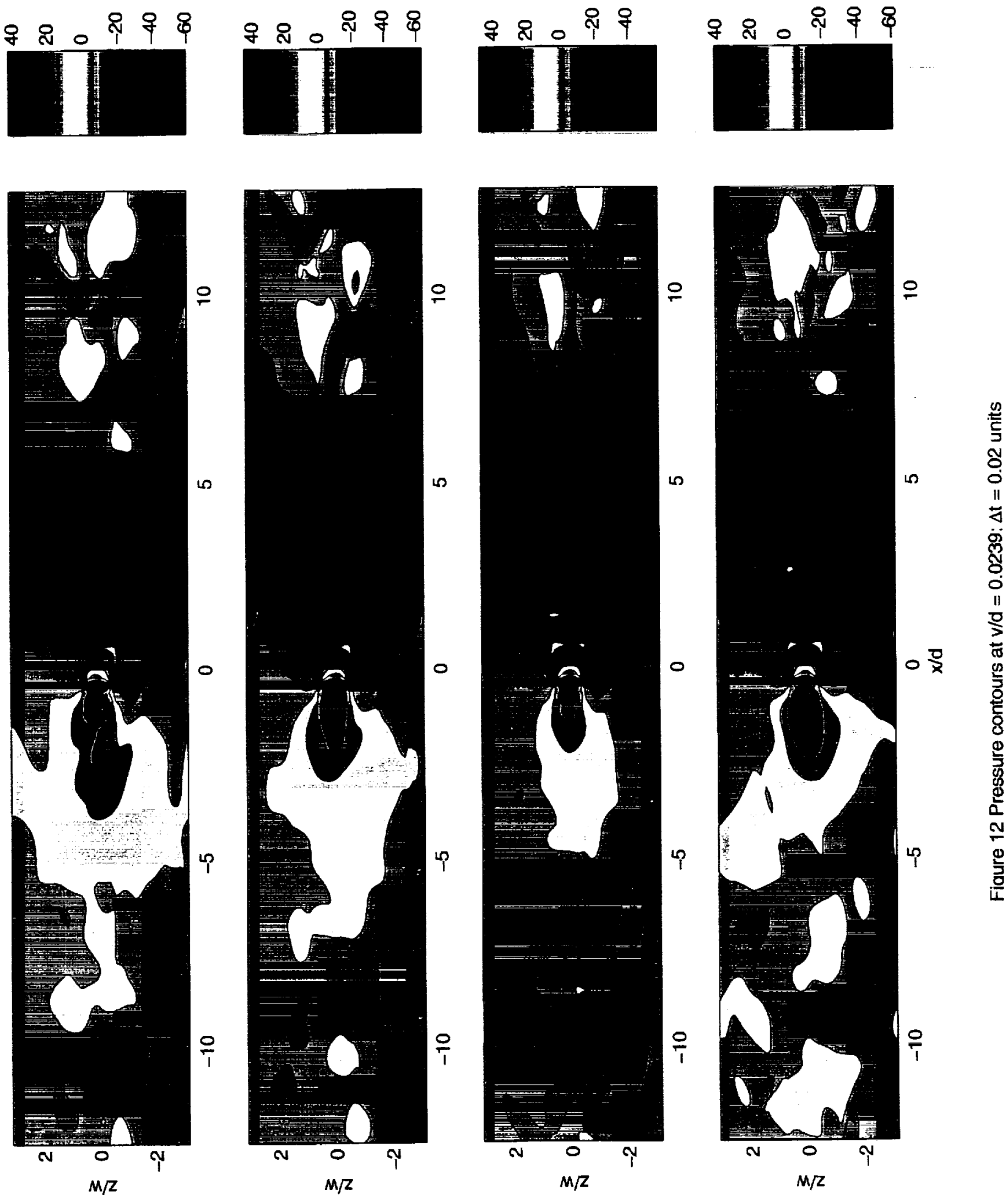

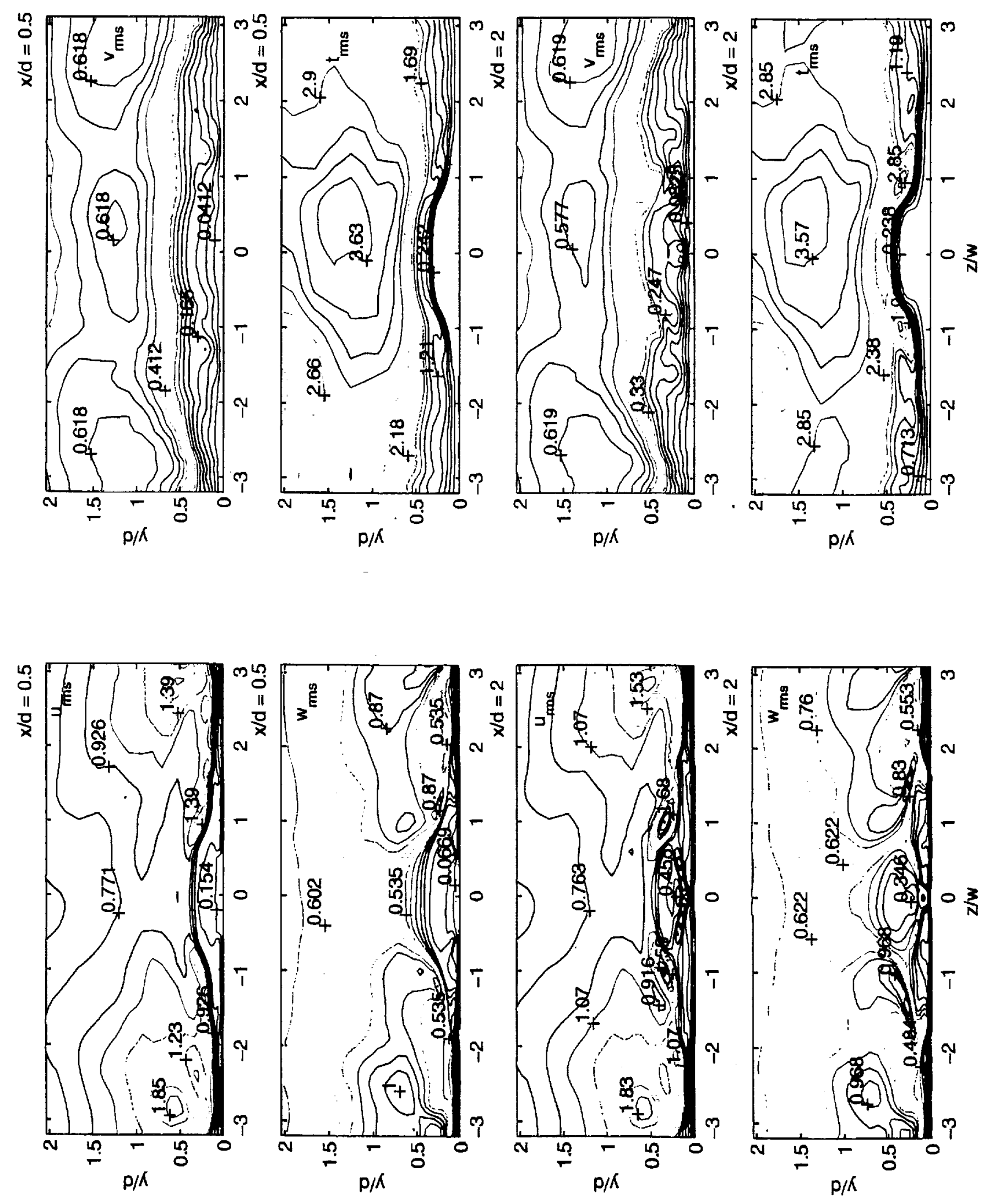


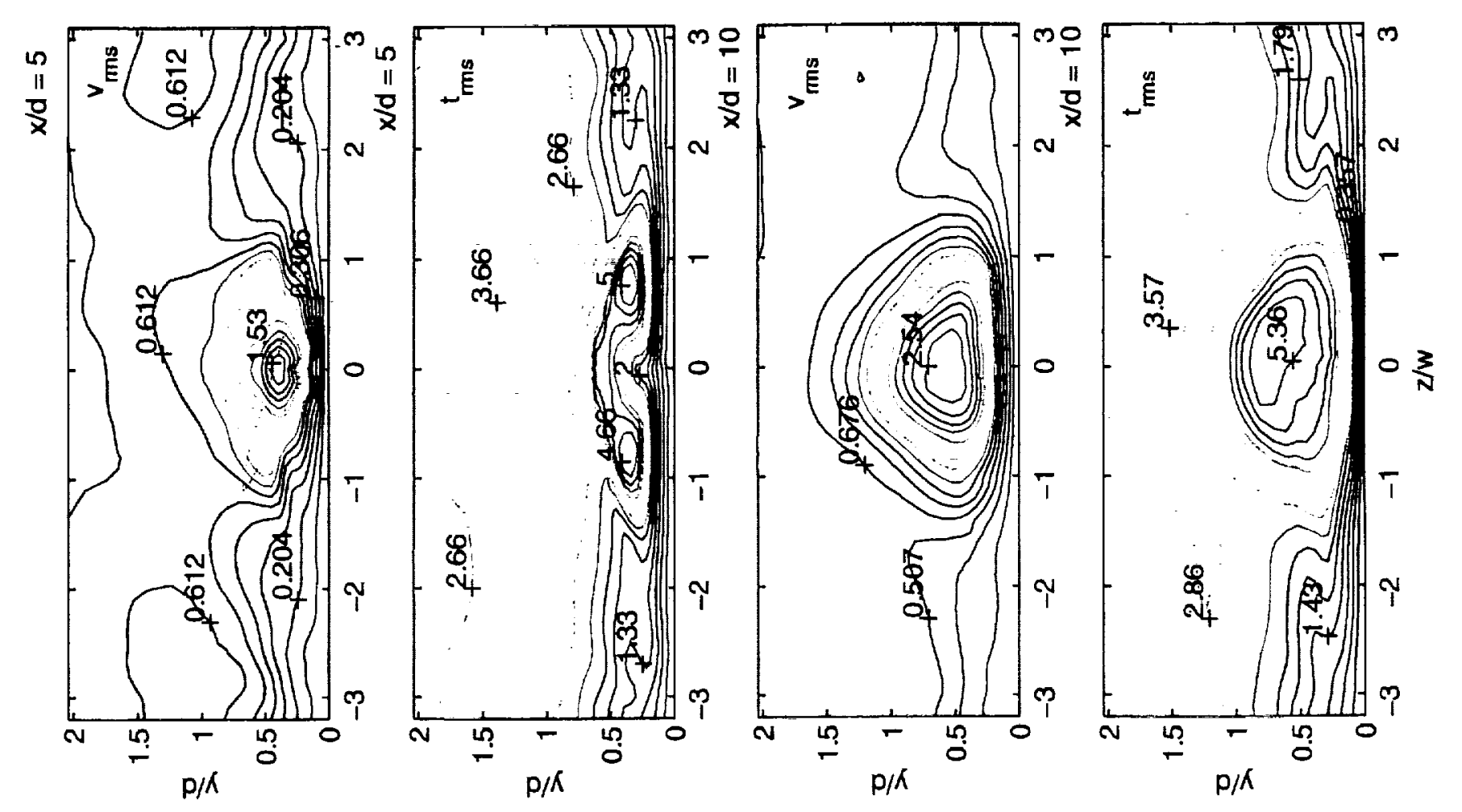

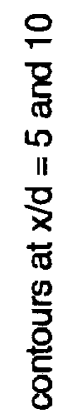
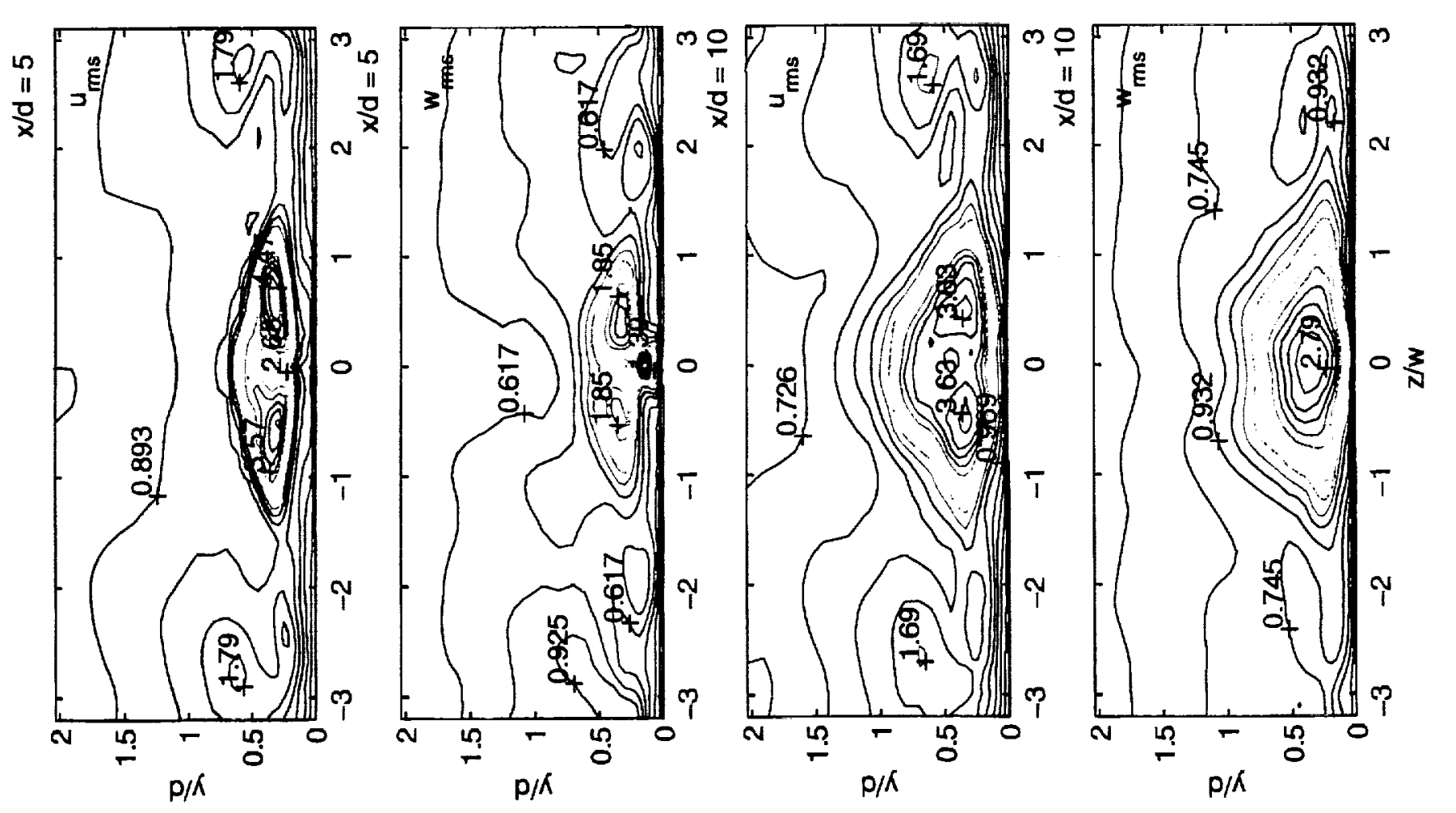


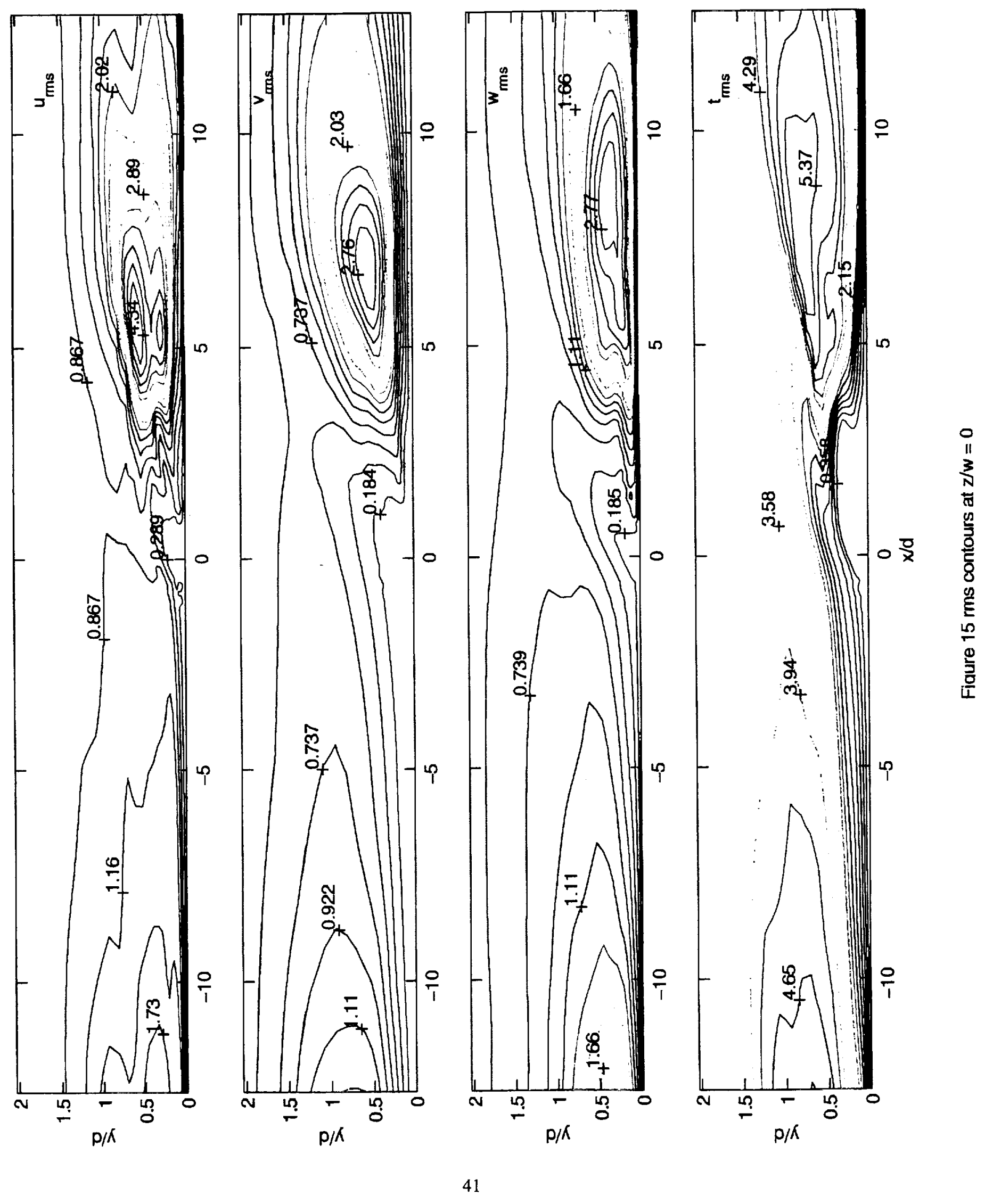



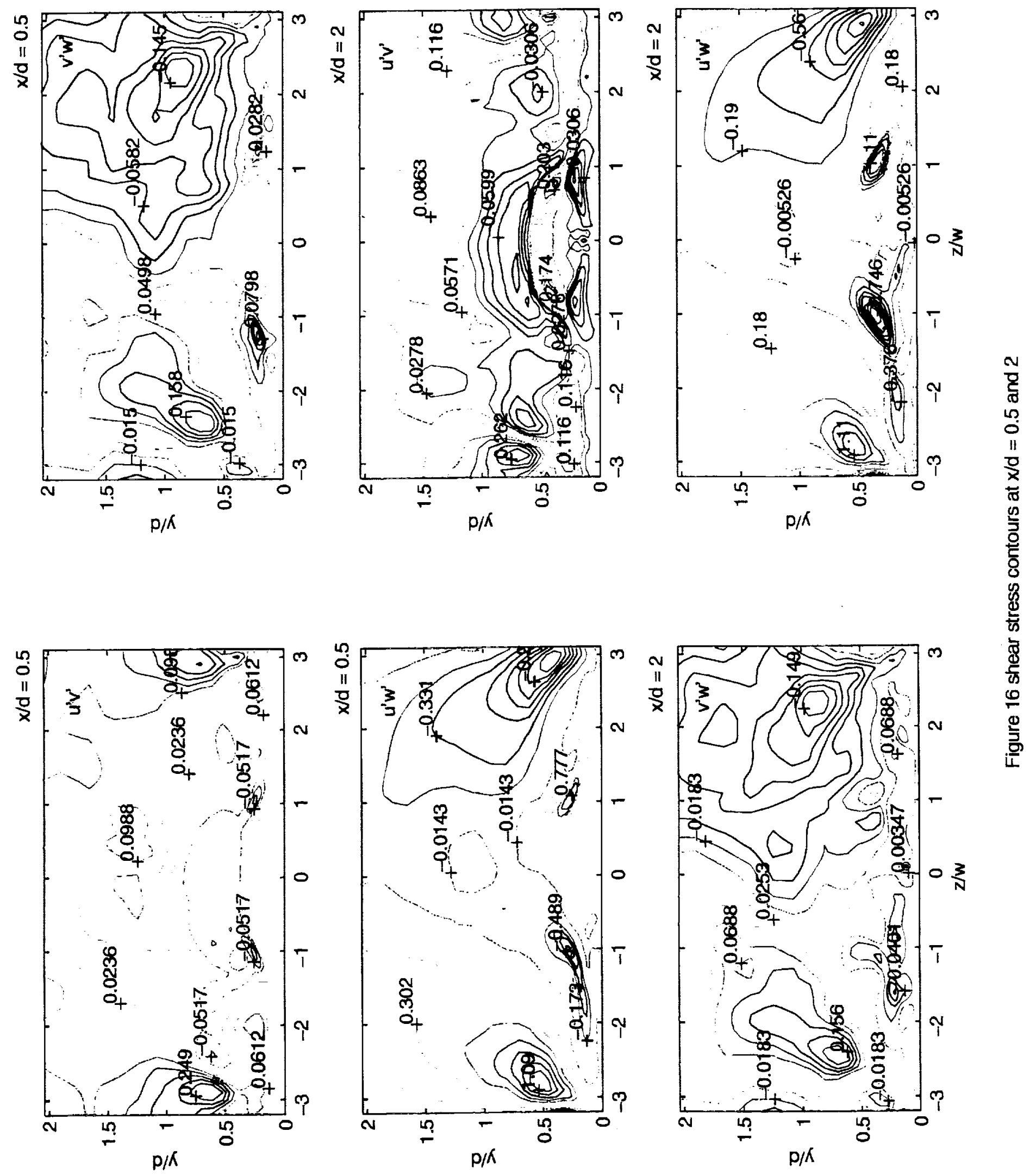

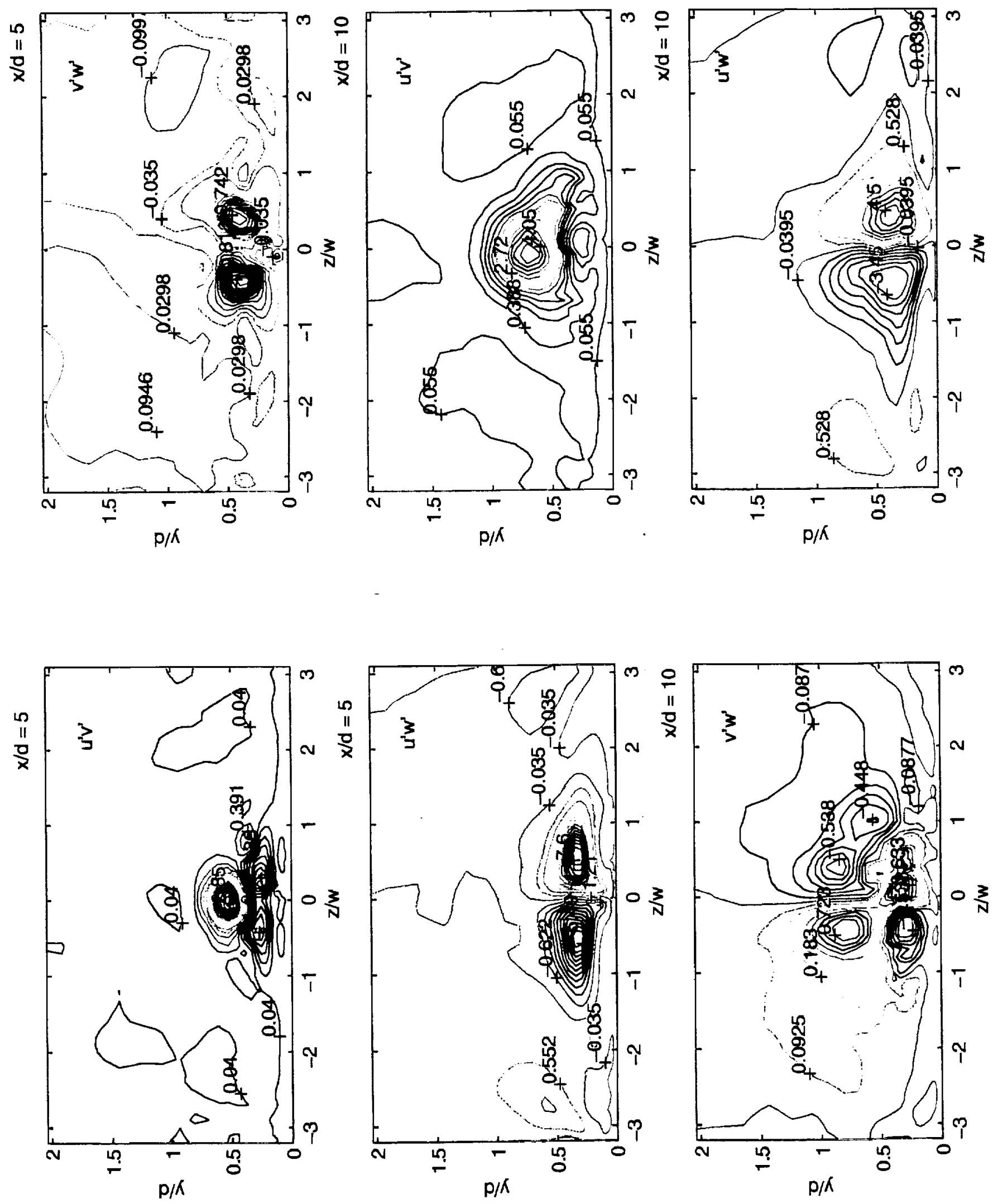

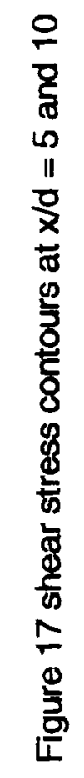




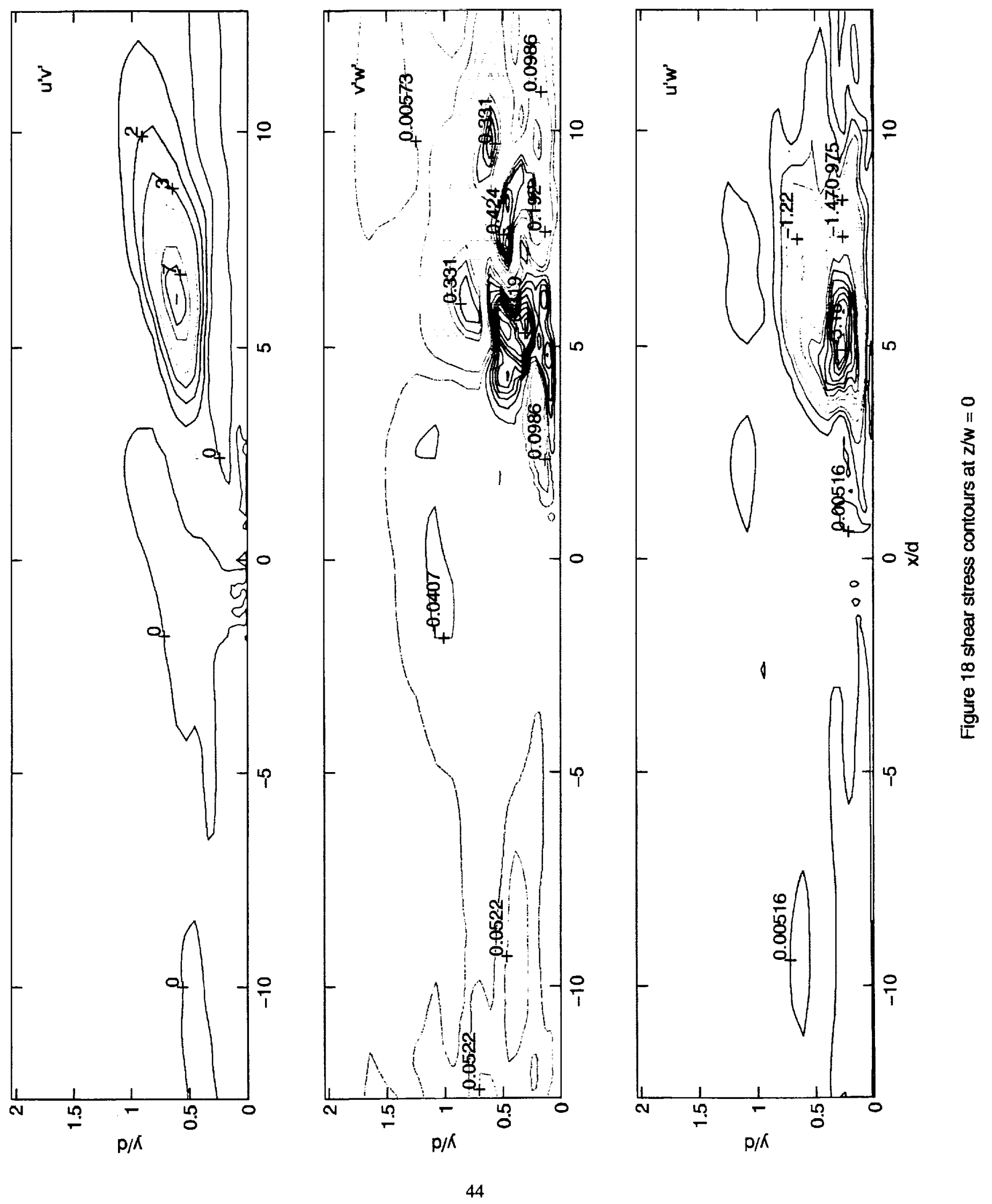



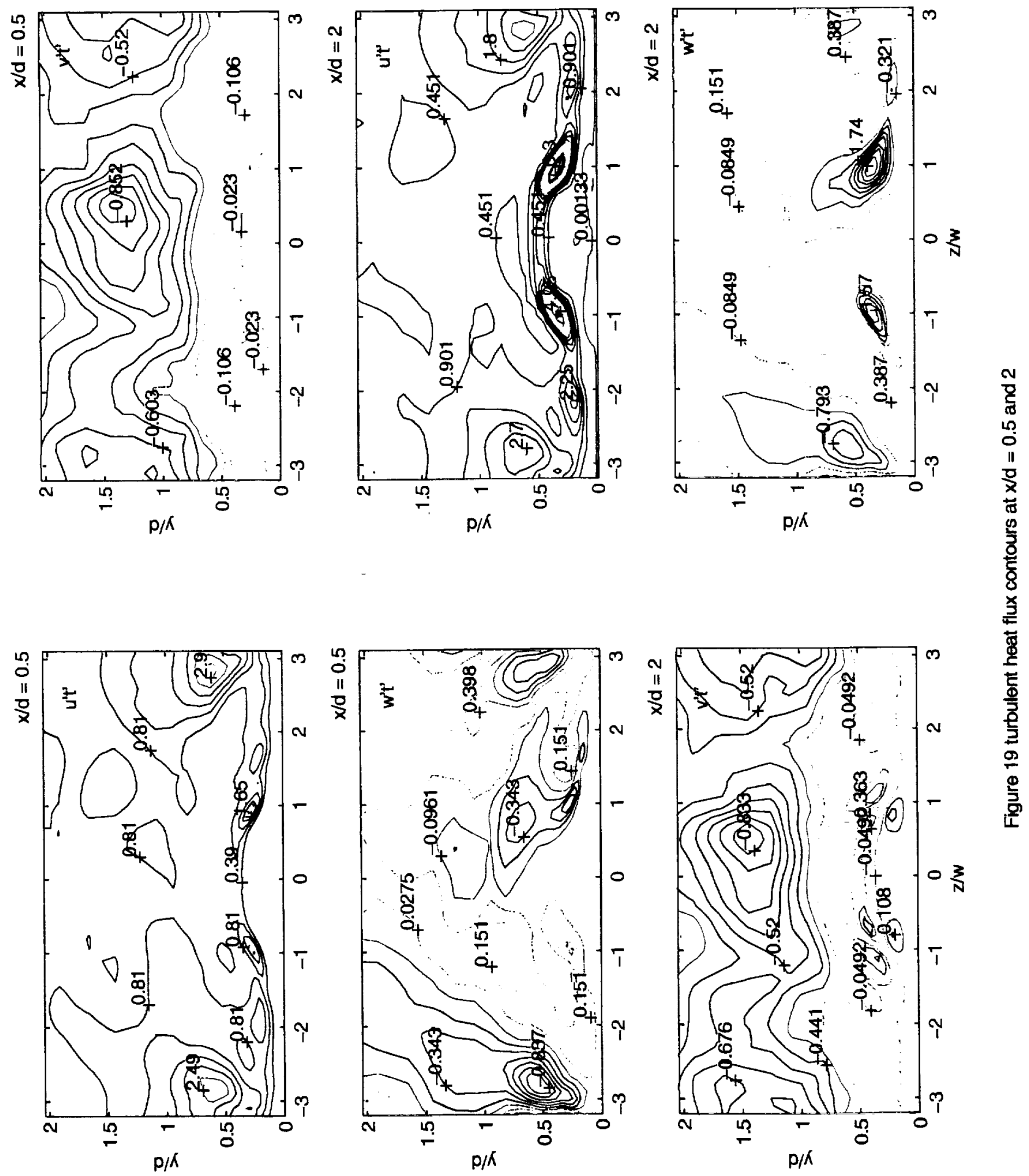

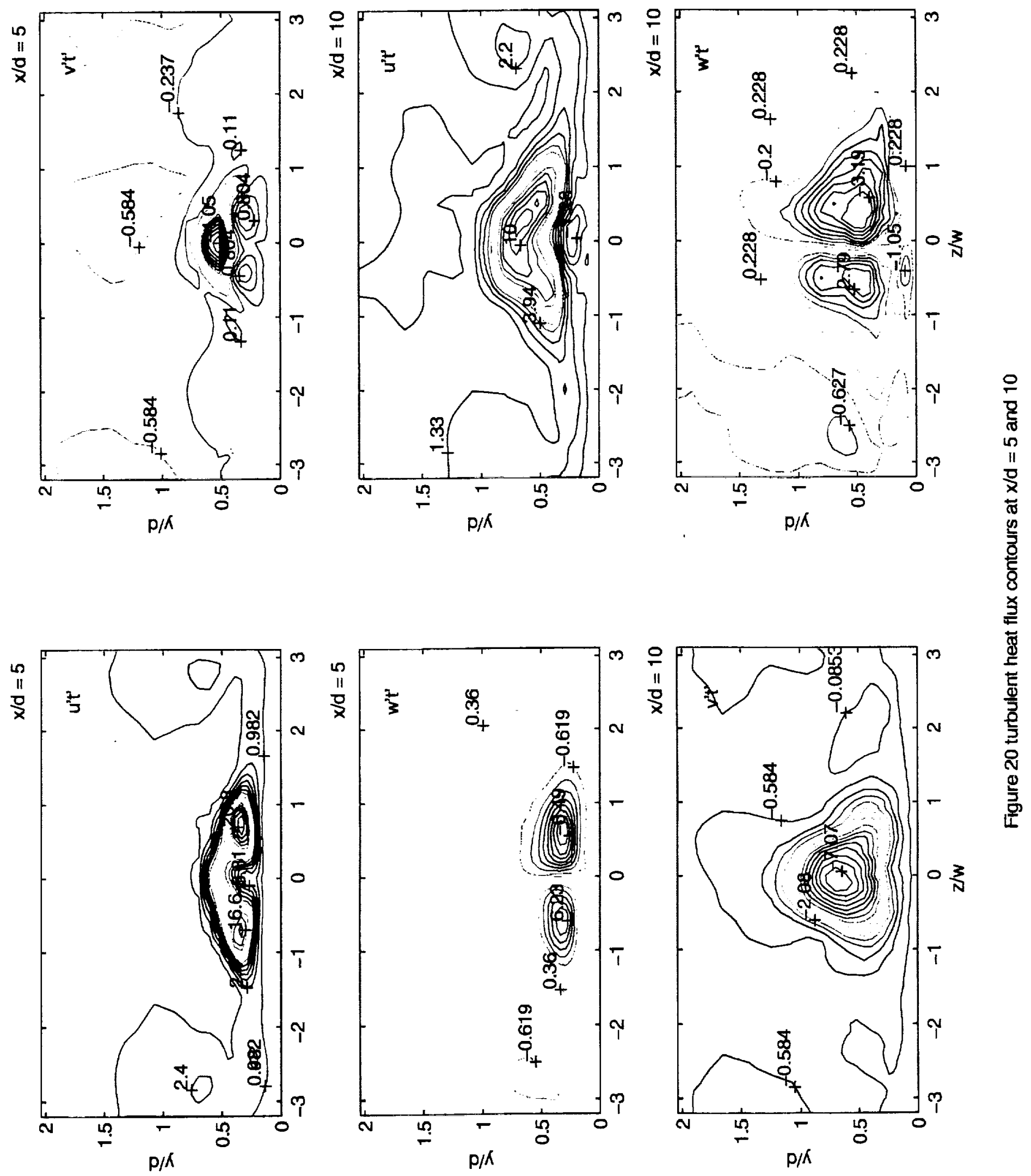

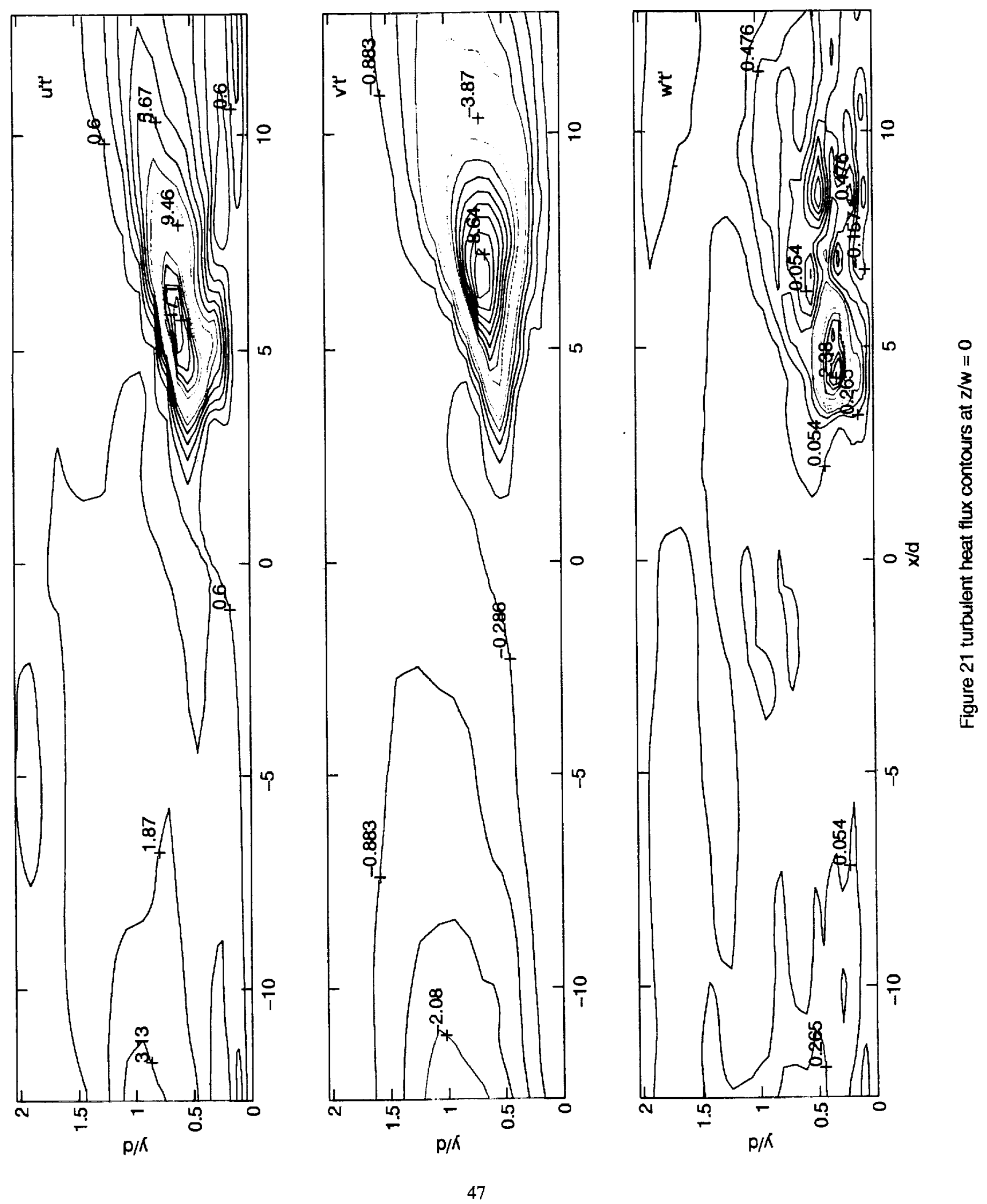


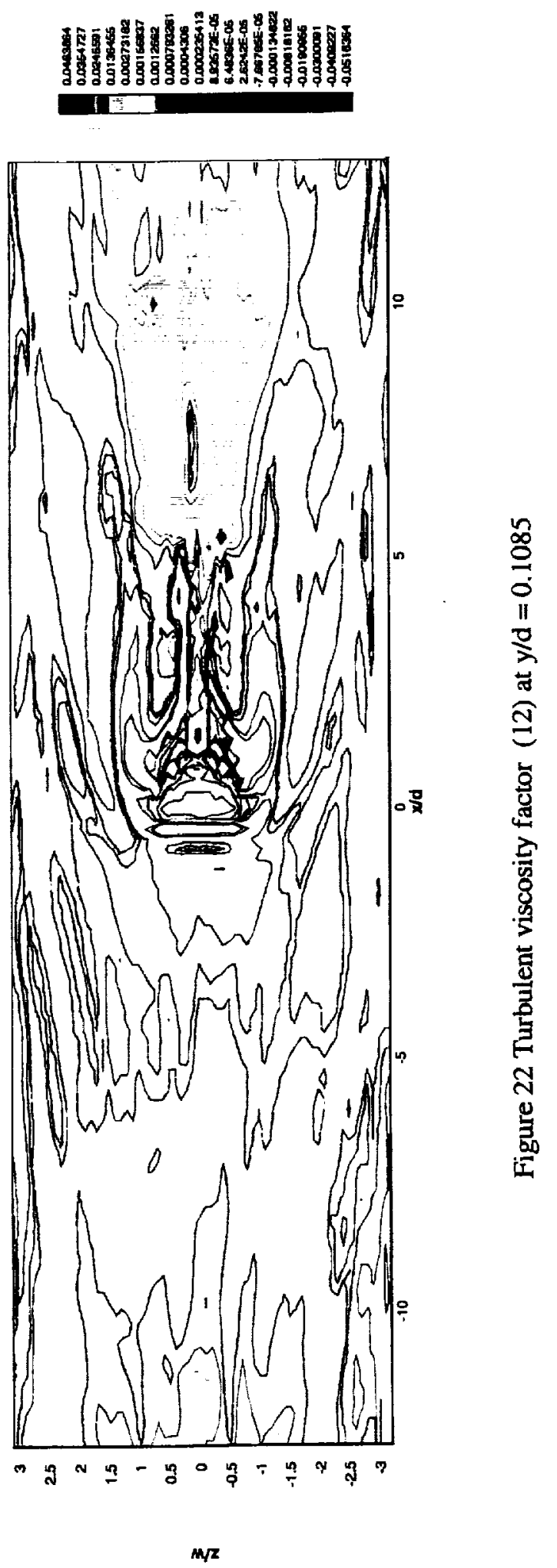



Public reponting burden for this collection of information is estimated to average 1 hour per response, including the time for reviewing instructions, searching existing data sources, gathering and maintaining the data needed, and completing and reviewing the collection of intormation. Send comments regarding this burden estimate or any other aspect of this gathering and maintaining daction Davis Highway. Suite 1204, Arlington, VA 22202-4302, and to the Otlice of Management and Budget, Paperwork Reduction Project (0704-0188), Washington, DC 20503.

\begin{tabular}{|l|l|l|} 
1. AGENCY USE ONLY (Leave blank) & 2. REPOAT DATE & 3. REPORT TYPE AND DATES COVERED
\end{tabular}

\begin{tabular}{|r|r|r|} 
October 1998 & Final Contractor Report
\end{tabular}

4. TITLE AND SUBTITLE

5. FUNDING NUMBERS

Direct Numerical Simulation of a Coolant Jet in a Periodic Crossflow

6. AUTHOR(S)

WU-538-12-10-00

NAG3-1641

Chirdeep Sharma and Sumanta Acharya

7. PERFORMING ORGANIZATION NAME(S) AND ADDRESS(ES)

Louisiana State University

Mechanical Engineering Department

8. PERFORMING ORGANIZATION

REPORT NUMBER

Baton Rouge, Louisiana 70803

E-11402

9. SPONSORING/MONITORING AGENCY NAME(S) AND ADDRESS(ES)

National Aeronautics and Space Administration

Lewis Research Center

Cleveland, Ohio 44135-3191

10. SPONSORING/MONITORING

AGENCY REPORT NUMBER

NASA CR-1998-208674

11. SUPPLEMENTARY NOTES

Prepared for the 1998 International Mechanical Engineering Congress and Exposition sponsored by the American Society of Mechanical Engineers, Anaheim, California, November 15-20, 1998. Project Manager, Chi Wang, Turbomachinery and Propulsion Systems Division, NASA Lewis Research Center, organization code 5820, (216) 433-5865.

12a. DISTRIBUTIONAVAILABILITY STATEMENT

12b. DISTRIBUTION CODE

Unclassified - Unlimited

Subject Categories: 02 and 34

Distribution: Nonstandard

This publication is available from the NASA Center for AeroSpace Information, (301) 621-0390.

13. ABSTRACT (Maximum 200 words)

A Direct Numerical Simulation of a coolant jet injected normally into a periodic crossflow is presented. The physical situation simulated represents a periodic module in a coolant hole array with a heated crossflow. A collocated finite difference scheme is used which is fifth-order accurate spatially and second-order accurate temporally. The scheme is based on a fractional step approach and requires the solution of a pressure-Poisson equation. The simulations are obtained for a blowing ratio of 0.25 and a channel Reynolds number of 5600 . The simulations reveal the dynamics of several large scale structures including the Counter-rotating Vortex Pair (CVP), the horse-shoe vortex, the shear layer vortex, the wall vortex and the wake vortex. The origins and the interactions of these vortical structures are identified and explored. Also presented are the turbulence statistics and how they relate to the flow structures.

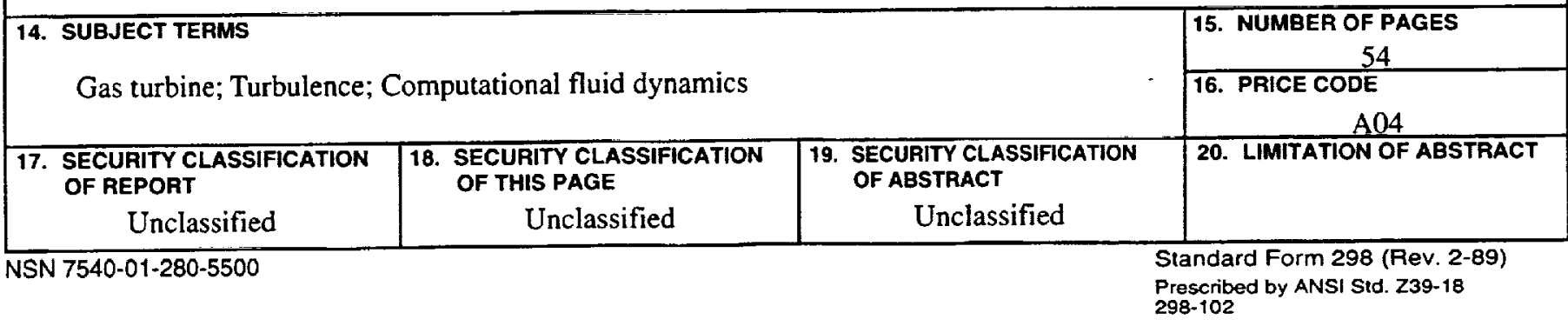

